\title{
Incoherent effect of space charge and electron cloud
}

\author{
G. Franchetti and I. Hofmann \\ GSI, Darmstadt, D-64291 Darmstadt, Germany \\ W. Fischer \\ Brookhaven National Laboratory, Upton, New York 11973, USA \\ F. Zimmermann \\ CERN, CH-1211 Geneva 23, Switzerland \\ (Received 23 April 2009; published 14 December 2009)
}

\begin{abstract}
Trapping by resonances or scattering off resonances induced by space charge (SC) or electron cloud (EC) in conjunction with synchrotron motion can explain observations of slow beam loss and emittance growth, which are often accompanied by changes in the longitudinal beam profile. In this paper we review the recent progress in understanding and modeling of the underlying mechanisms, highlight the differences and similarities between space charge and electron cloud, and discuss simulation results in the light of experimental observations, e.g., at GSI, CERN, and BNL. In particular, we address the role of the pinched electrons and describe in detail the complexity of the electron pinch formation. We present simulation results within a dipole or in a field-free region of the beam pipe, which reveal the morphology and main features of this phenomenon, explain the physical origin of the complex electron structures like stripe in either field configuration, and discuss the dependence on some key parameters.
\end{abstract}

DOI: 10.1103/PhysRevSTAB.12.124401

PACS numbers: 29.27.Bd, 05.45.Pq, 52.35.Qz, 52.40.Mj

\section{INTRODUCTION}

The term "incoherent," describing the effects of space charge in a 2D beam, is normally used to mean effects due to the incoherent tune shift [1] of each beam particle, which is created by the beam self-force together with image forces due to the beam-pipe boundary. By contrast, "coherent" space-charge effects in the transverse plane are commonly associated with the collective beam response to the beam perturbations [2,3], and in the absence of these coherent effects no relevant emittance growth is expected to occur due to the intrinsic beam nonlinearities alone.

The only exception arises when the beam traveling through a circular accelerator is affected by the lattice optics and experiences an $s$-dependent change in the space-charge force via envelope modulation. This beam behavior drives several waves of nonlinear force along the machine responsible for the excitation of new resonances: such resonances are called "structure" resonances.

The interplay of the coherent tune shift with latticedriven resonances or with structure resonances is then an essential ingredient for the correct identification of the tunes where a resonant effect will occur [4]. Past studies on this topic were mainly performed considering 2D beams. But the request for long-term storage of high intensity bunches in circular accelerator motivated the study of the full 3D problem. The beam dynamics of a bunch is approximated by partially decoupling the dynamics of the transverse-longitudinal planes: the synchrotron motion is considered, in first approximation, independent. An important effect of the synchrotron motion on the particles in a bunch is to advance them longitudinally and via space charge induce a transverse tune modulation at a frequency twice the synchrotron frequency. In the CERN proton synchrotron, this mechanism was tested under controlled experimental conditions [5]. It was found that the beam response and beam loss are consistent with the numerical modeling. The underlying mechanism for this beam response relies on the space-charge transverse tune modulation for inducing a periodic resonance crossing. In this beam dynamics regime trapping/scattering of beam particles into the resonance creates a complex diffusion-type dynamics which becomes evident only after many synchrotron oscillations. Only the particles which cross the resonance are subjected to trapping/scattering and this condition of "resonance crossing" depends on the initial particle invariants $\epsilon_{x}, \epsilon_{y}, \epsilon_{z}$, the space-charge tune-shift $\Delta Q_{x, s c}$, and the working point $\left(Q_{x 0}, Q_{y 0}\right)$. In Ref. [5] it is shown that the maximum amplitude a particle can reach depends on the distance from the resonance approximately as $\sim 1 /\left(Q_{x}-Q_{x, \text { res }}\right)$. This dependence creates two regimes: a beam loss regime for tunes located in the proximity of the resonance (above), and a neighboring emittance growth regime (no beam loss). In Ref. [6] the role of the transverse tune dependence induced by space charge is discussed for a Gaussian stationary bunched beam. The fraction of particles to be trapped/scattered is estimated as $\Delta N / N \sim\left(Q_{x}-Q_{x, \text { res }}\right) / \Delta Q_{x, \text { sc }}$. As only particles with large synchrotron amplitude will span the full space-charge tune spread and therefore may reach a large transverse amplitude, the beam loss will shorten the bunch 
length [7]. Recently, also the role of chromaticity in the 3D high intensity bunched beams was explored and it was found that it enhances beam loss bringing the numerical results closer to the experimental findings [8].

In proton or ion storage rings operating with trains of closely spaced "short" bunches, such as LHC or RHIC, an electron cloud builds up over the passage of many bunches. A similar buildup repeats from turn to turn as the same bunch train passes again through the same electron cloud (EC) region. During the passage of each individual bunch in the train, the EC near the beam "pinches," under the influence of the locally large electric field of the bunch, and close-by electrons are attracted towards the beam center. In this paper we do not further discuss the process of electroncloud buildup due to a bunch train, but instead we study the less explored incoherent effect of an existing preformed electron cloud (generated by preceding bunches) on one passing bunch. The average density of the preformed electron cloud would typically be saturated, where the saturation is normally due to the effect of the electron cloud's electric self-field. The electron-cloud buildup itself has been studied for many years, and numerous simulation codes and analytical models are available to model it. In the following, we concentrate on the (incoherent) interaction, with the preformed cloud, of a single bunch in a train of bunches passing through this same cloud again and again, on successive turns.

The presence of the electron cloud in proton machines has long been associated with the creation of instabilities $[9,10]$. The interaction of localized electrons with proton beams is very complex in terms of formation and dynamics: when a proton bunch passes through a localized electron cloud it causes a pinch of the electron cloud itself $[11,12]$. The idea that the pinched electron cloud may also give rise to incoherent effects on the proton beam has been around for several years. At the ICFA-HB2004 workshop, the analogy with space-charge-induced trapping phenomena was brought into the discussion. The essential key suggesting a similarity with space charge is the correlation of the amount of pinch with the extent of the bunch that has passed through the EC. This correlation creates a dependence of the pinch experienced by a bunch particle and its longitudinal position inside the bunch at the time of passage through the EC [13]. In this dynamics the electrons are the weak "beam" subject to large variations in density, which however may "resonantly" feed back on the strong main beam. For a bunch longer than the EC extension, the EC pinch occurs several times for the same electrons [11] according to the bunch charge density and sizes. The possibility of trapping/scattering induced by pinched EC was shown in [14]. There, a simplified model of the pinching electron cloud was employed, considering an electron density inside the beam which was growing linearly from the bunch head to the bunch tail. This model revealed that a slow emittance growth could be created similarly to what happens with space charge. Clearly, the prediction capability of such a type of model is based on the accurate modeling of the pinched EC. Simulations in fact show that the EC pinch progresses and evolves as the bunch passes through the EC, and that it exhibits a complicated time dependent EC morphology with "rings" [12]. A previous attempt to model the effect of such rings is reported in Ref. [15] where a one-dimensional model was studied.

Here we extend the EC modeling to two-dimensional EC "rings," and compare the resulting EC effect on the bunch dynamics with that induced only by the beam space charge (SC). The electron space charge is neglected in the modeling of the pinch, since the density of beam particles inside the bunch is typically many orders of magnitude higher than the local electron-cloud density prior to the bunch passage. One reason for this order-of-magnitude difference is that the electron cloud is distributed over the entire beam pipe, while the beam only occupies a small area of the pipe, both transversely and longitudinally. Nevertheless, the effect of the electron-cloud space charge, together with an initial electron velocity spread, may ultimately limit the local electron peak density of the pinch occurring inside the bunch [13].

\section{ELECTRON-CLOUD DYNAMICS}

When a proton or positron bunch passes through an electron cloud generated by preceding bunches, the cloud electrons are attracted towards the transverse center of the bunch or "pinched," resulting in regions of high electron density inside the bunch. This electron-cloud "pinch" gives rise to an incoherent betatron tune shift, which varies with the longitudinal position and with transverse amplitude. Combined with synchrotron motion and together with the nonuniform distribution of the electron cloud around a storage ring [in the super proton synchrotron (SPS), for example, the electron cloud builds up preferentially inside the dipole magnets [16] ], this tune shift in turn leads to the excitation of betatron and synchrobetatron resonances $[14,17]$, as well as to "scattering" off these resonances [14]. For the LHC proton beam in the proton synchrotron (PS), SPS, and LHC itself, these effects can be significant [18]. Some of their characteristics resemble space-charge phenomena $[14,15,19]$.

Early models of the electron pinch assumed an electron density, or tune shift, that linearly increases along the bunch. Simulations and analytical treatments show that in reality, due to the nonlinear oscillation of electrons in the bunch potential, stripes of high-density form close to the center of the bunch and then propagate outwards [13]. The presence of a dipole magnetic field restricts the horizontal motion of the electrons, and can lead to the appearance of different, "elliptical" stripes, that again start at the transverse bunch center and later shift outwards [20]. Recent studies using a refined pinch model with stripes have uncovered a complex phase-space structure, indicating 
the possibility of larger beam losses and stronger emittance growth than previously anticipated [15,21].

\section{A. Electron motion}

If the transverse beam size is much smaller than the vacuum chamber, we can approximate the electron-cloud density in the vicinity of the beam prior to a bunch arrival by a uniform distribution. Under the influence of the electric field of the bunch, the electrons of the initially uniform cloud are perturbed and develop a structure with local density enhancements.

The electron motion in the bunch potential is characterized by the linear oscillation frequencies of electrons close to the transverse center of the beam, $\omega_{e ; x, y}$. In the absence of an external magnetic field and for a round bunch the frequency is the same in both planes, and, assuming a transverse Gaussian density with rms size $\sigma_{r}$, equal to

$$
\omega_{e}(z)\left[\mathrm{m}^{-1}\right] \equiv \frac{\sqrt{\lambda(z) r_{e}}}{\sigma_{r}},
$$

for an arbitrary longitudinal line particle density $\lambda(z) . r_{e}$ is the classical radius of the electron. Introducing the radial coordinate $r=\sqrt{x^{2}+y^{2}}$, and its normalized counterpart $\tilde{r} \equiv r / \sigma_{r}$, the electron equation of motion is

$$
\frac{d^{2} \tilde{r}}{d z^{2}}+\omega_{e}^{2}(z) \tilde{r}=-\frac{\omega_{e}^{2}(z)}{\tilde{r}}\left[2\left(1-e^{-\left(\tilde{r}^{2} / 2\right)}\right)-\tilde{r}^{2}\right],
$$

where the left-hand side represents the linear oscillation at small amplitudes, and the right-hand side the nonlinear terms. We observe that $\omega_{e}(z)$ defines the scaling of the electron motion, so that, e.g., for a fixed longitudinal shape, doubling the bunch intensity is equivalent to halving the bunch length, or to shrinking the transverse beam sizes by $\sqrt{2}$.

Note that Eq. (2) neglects the electric self-force of the electrons. This approximation affects the maximum peak electron densities that can be reached during the pinch process. Namely, when many pinching electrons simultaneously approach the longitudinal axis, the repulsive force exerted by the electrons on themselves compensates some of the attractive electric force exerted by the proton bunch, which smears out the electron arrival times on axis, slightly shifts the location of the pinch center, and reduces the microscopic peak density. In the analysis carried out in this paper we neglect this self-force effect on the basis that the initial electron density is much lower than the beam ion density inside the bunch, so that the effective shift of the pinch center on axis is small with respect to the distance of two consecutive pinches, and, in addition, that our coarsegrain model described later does not allow for unphysical, locally infinite electron densities, but spreads out the electrons over a transverse distance $\Delta R$ as in Eq. (18).
If the bunch is not round, the horizontal and vertical oscillation frequencies differ, and a second parameter or function is necessary to characterize the pinch.

In the case of a strong dipole field oriented in the vertical direction, we can consider the electrons' horizontal position as frozen. The vertical force yields the equation of motion,

$$
\frac{d^{2} \tilde{y}}{d z^{2}}+\omega_{e}^{2}(z) \tilde{y}=-\frac{\omega_{e}^{2}(z) \tilde{y}}{\tilde{r}^{2}}\left[2\left(1-e^{-\left(\tilde{r}^{2} / 2\right)}\right)-\tilde{r}^{2}\right],
$$

where we have introduced a normalized vertical coordinate $\tilde{y} \equiv y / \sigma_{r}$, and again assumed a round beam $\left(\sigma_{r} \equiv \sigma_{x}=\right.$ $\left.\sigma_{y}\right)$. Also here $\omega_{e}(z)$ characterizes the electron motion completely, via Eq. (3).

For comparing results it is convenient to introduce the linear oscillation phase

$$
\phi_{e}(z) \equiv \int_{-\infty}^{z} \omega_{e}\left(z^{\prime}\right) d z^{\prime}
$$

which for two specific longitudinal profiles translates to

$$
\phi_{e}(z)=\frac{\sqrt{r_{e} N_{b}}}{\sigma_{r}} \times \begin{cases}\frac{\sqrt{\sigma_{z} \pi}}{(2 \pi)^{1 / 4}}\left[1+\operatorname{erf}\left(\frac{z}{2 \sigma_{z}}\right)\right] & \text { (Gaussian) } \\ \sqrt{\frac{l_{b}}{2}}\left(1+\frac{z}{l_{b}}\right) & \text { (uniform) }\end{cases}
$$

where $l_{b}$ is the half length of the bunch for the case of the uniform longitudinal profile, and the variable $z$ varies in the range $-l_{b}<z<l_{b}$.

\section{B. Simulation parameters}

We integrate Eq. (2) or (3) with the Runge-Kutta method and track the evolution of about $5 \times 10^{5}$ macroelectrons, which are launched evenly distributed in the transverse space on a wide circular area of radius $10 \sigma_{r}$ with zero initial velocity. The integration step is taken to be $d s=$ $0.006 \sigma_{z}$. The initial electron energy of a few electron-volt can be neglected, since the typical electron kinetic energy acquired during the pinch is much larger, of order $m_{e}\left(c \omega_{e} \sigma_{r}\right)^{2} / 2$, where $m_{e}$ denotes the electron mass and $c$ the speed of light. For simplicity, we will consider only circularly symmetric bunches, for which the electron motion is described by Eq. (2) or (3). The bunch parameters in our simulation represent the LHC beam at injection: We consider $N_{b}=1.15 \times 10^{11}$ protons per bunch, with transverse rms size $\sigma_{x, y}=0.88 \mathrm{~mm}$ and rms bunch length $\sigma_{z}=$ $11.4 \mathrm{~cm}$. The transverse beam distribution is taken to be Gaussian; in the longitudinal plane we choose either a Gaussian or a uniform shape. The zero of the longitudinal coordinate $z$ coincides with the bunch center.

\section{Stripe structure}

Figure 1 presents the simulated density enhancement in the $x-z$ plane at $y=0$ (left pictures) and also in a parallel plane with $2 \sigma_{r}$ vertical offset (right pictures). The top 

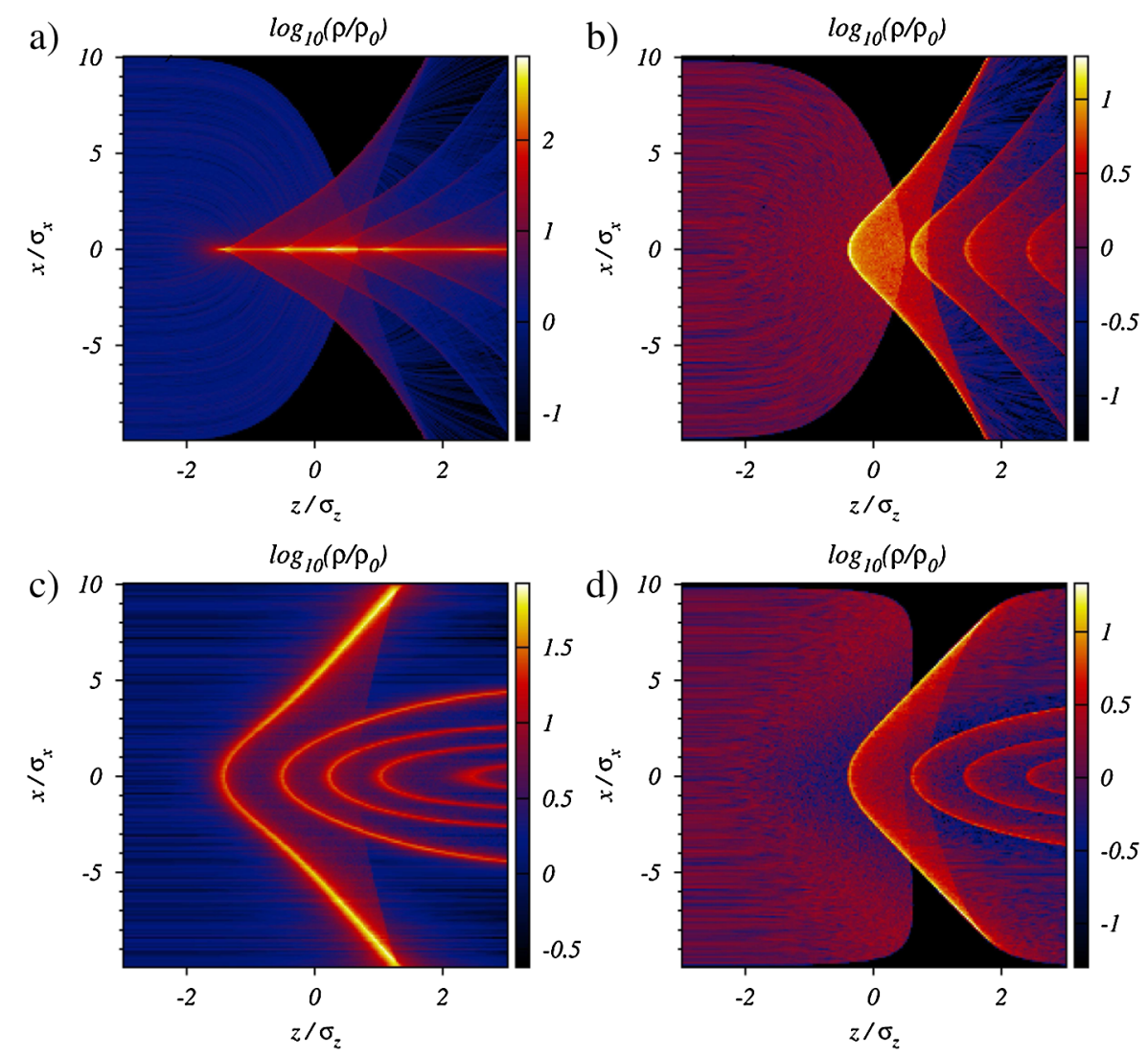

FIG. 1. (Color) Electron density enhancement in the $x-z$ plane at $y=0$ (left) and $y=2 \sigma_{r}$ (right) in a field-free region (top) and in a dipole (bottom), for a Gaussian bunch.

pictures show results for a field-free region, the bottom ones for a dipole field. In all cases, about four stripes emerge during the passage of the bunch. For the fieldfree region the electron density at the center of the bunch, at $y=0$, becomes very high (note the different logarithmic density scale). For a plane with vertical offset, $y=2 \sigma_{y}$, the stripe structure becomes clearly visible also in the fieldfree case. Figure 2 shows the corresponding density in the $x-y$ plane at the longitudinal position $z=1 \sigma_{z}$.
Analyzing these data, the left picture of Fig. 3 presents the simulated vertical position of the outermost (horizontal) stripe as a function of its horizontal position. The stripe for the dipole is almost of the same round circular shape in the $x-y$ plane as the one without field and only slightly flattened. The right picture shows that the density in the stripe hardly varies with horizontal position, both without field and in a dipole.
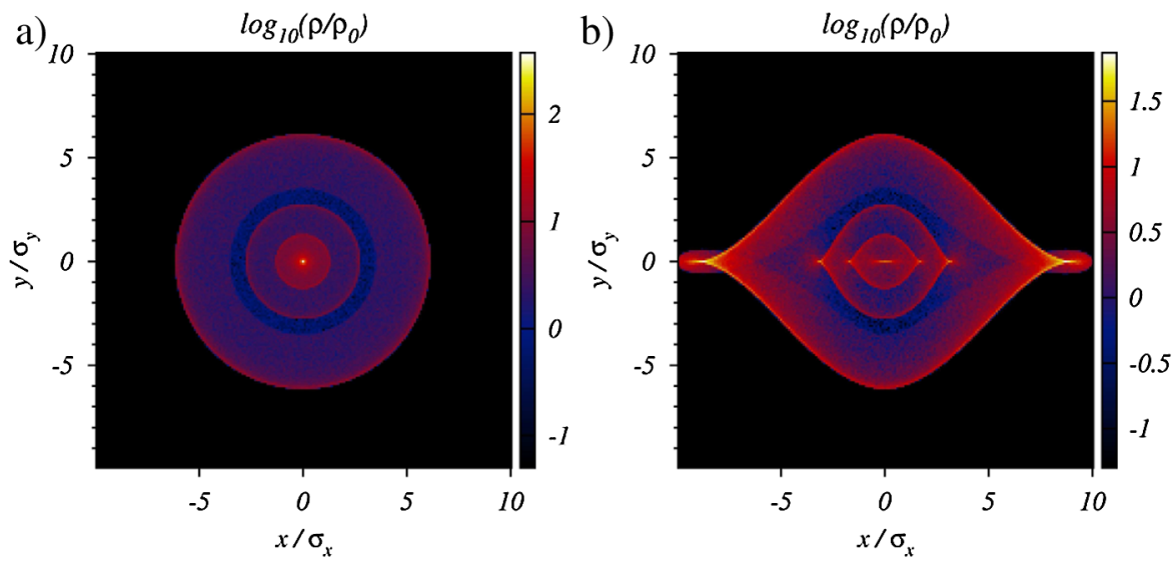

FIG. 2. (Color) Electron density enhancement in the transverse plane (in units of proton bunch sizes) at $z=1 \sigma_{z}$ without field (left) and in a dipole (right). 

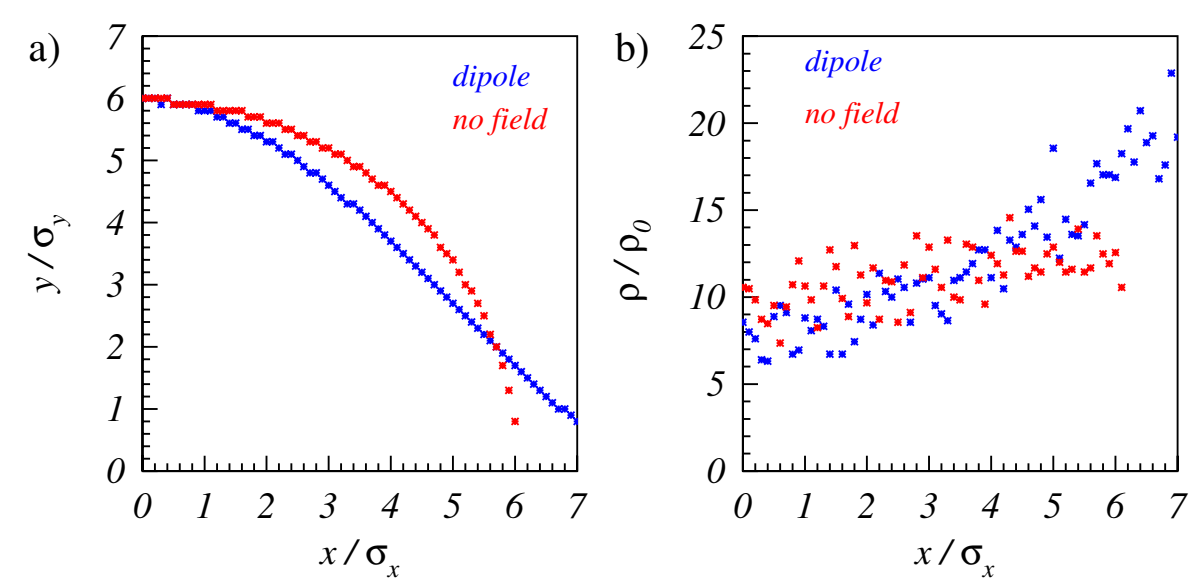

FIG. 3. (Color) Vertical vs horizontal position of the outermost stripe at $z=1 \sigma_{z}$, i.e., $\phi_{e} /(2 \pi) \simeq 1.87$ (left), and the peak density in this stripe vs the horizontal position (right), comparing a field-free region and a dipole magnet.

Simulations were also performed for a longitudinal uniform bunch profile with a full bunch length chosen equal to $2 l_{b}=6 \sigma_{z}$. Figure 4 demonstrates that, when plotted as a function of $\phi_{e}$ instead of $z$, the spatial distribution and magnitude of the density enhancement are similar, albeit not fully identical, for the Gaussian and uniform longitudinal profiles. Always a new "stripe" emerges on axis
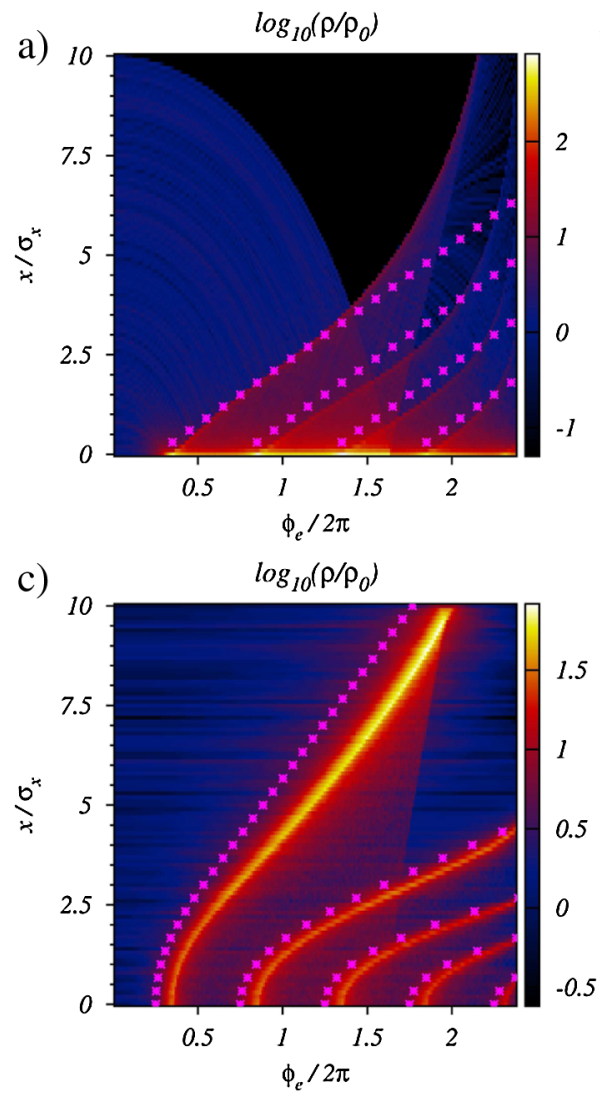

roughly at every half period of linear oscillation, starting from $\pi / 2$, i.e., at $\phi_{e}=\pi / 2,3 \pi / 2$, etc.

\section{Discussion}

Comparing the top and bottom pictures in Figs. 1 or 4, we notice that, while in a field-free region the electron density of a stripe decreases as the latter "moves" to larger
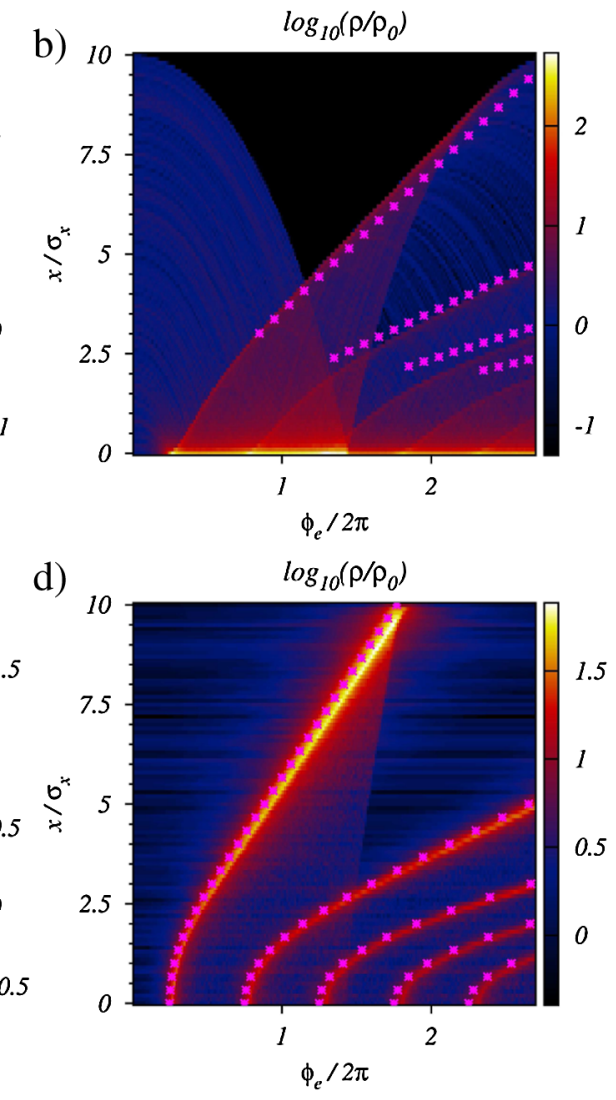

FIG. 4. (Color) Electron density enhancement in the $x-\phi_{e}$ plane, at $y=0$, with a Gaussian (left) or uniform longitudinal profile (right) without field (top) and in a dipole (bottom). Note the markers which on top which are obtained by Eq. (8) for (a), and Eq. (6) for (b). On the bottom pictures the markers are from Eq. (10). 


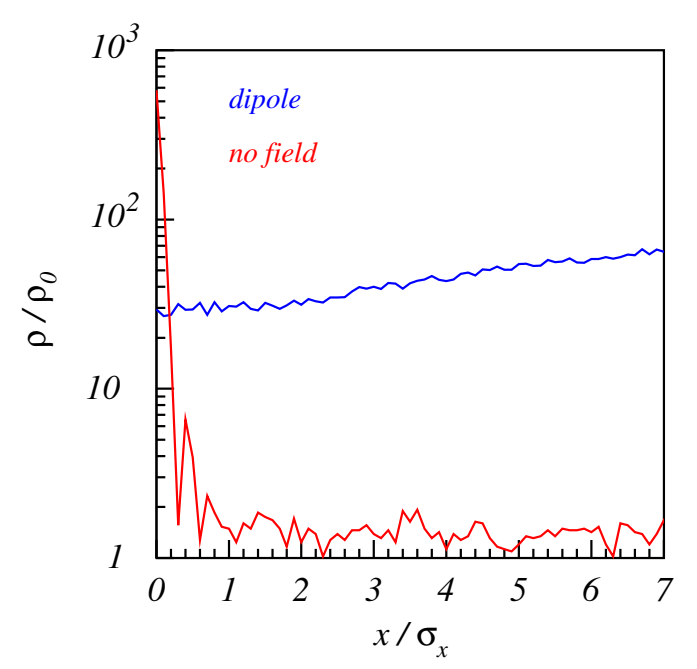

FIG. 5. (Color) Density enhancement in the outermost stripe as a function of horizontal stripe position, for a field-free region and a dipole.

amplitudes, in a dipole field the density increases instead. This is further illustrated in Fig. 5 which shows the simulated density in the outermost stripe, in the plane $y=0$, as a function of its horizontal position.

The physical origin of the stripe patterns differs for the field-free region and for the dipole field. Without magnetic field the electrons move radially, with dipole field vertically (or horizontally).

The physical origin of the enhanced region of electroncloud density, or "pinch," stems from the phenomenon of trajectory overlapping in physical space. For example, if the attractive force exerted by the protons on the electrons was exactly linear, i.e., proportional to $\tilde{r}$, then each electron would oscillate with exactly the same wavelength. In such a situation the trajectories of all electrons, which are taken to be at rest prior to the bunch passage, would all cross the longitudinal axis, defined by $\tilde{x}=\tilde{y}=0$, together after the first $\pi / 2$ of electron phase advance and again later on each following multiple of $\pi$. As all these electron trajectories cross in the same physical point (again neglecting the selfforce), the electron density is very high. The real force is approximately linear at the origin and many electrons, e.g., those which are initially located within the transverse rms beam size, still cross the longitudinal axis at about the same time, leading to the appearance of the "pinch."

The dynamics of the electrons as described by Eq. (2) contains an additional complexity: For electrons initially located near the transverse bunch axis, the motion is fairly linear as described by Eq. (2). Hence, they all oscillate in phase originating density peaks at $\tilde{x}=\tilde{y}=0$ as previously discussed. However, these electrons do not play a role in creating the electron-cloud structure far off the origin. This role is rather played by the electrons located initially far from the transverse origin. These electrons, in fact, undergo a highly nonlinear motion, and their oscillation wavelength depends on their initial amplitude (the equivalent to an amplitude-dependent detuning for beam, in the language of space charge). The larger their amplitude, the longer their wavelength is as result of the reduction of the average attractive force from the proton bunch. This particular feature is responsible for another type of "trajectory crossing," namely, one of neighboring electrons initially located at large amplitude. An electron initially located at the amplitude $\tilde{r}_{0}$ has a longer oscillation wavelength than a neighboring electron initially located at $\tilde{r}_{0}-d \tilde{r}_{0}$. Hence, in half an oscillation period, the trajectory of the electron located initially at $\tilde{r}_{0}$ will necessarily cross the trajectory of the electron which started at $\tilde{r}_{0}-d \tilde{r}_{0}$. In the same way at the crossing of physical trajectories at the origin, the crossing point of neighboring trajectories at large amplitudes defines a region of enhanced electron density and will occur close to the local turning point of the transverse electron oscillation, which happens every half a wavelength. Consequently, a local maximum in the electron density (the electron stripe) is found near the turning point of the transverse electron motion, i.e., around the point where the transverse electron speed is zero.

To estimate the amplitude of the turnover point, we may consider electrons which started their journey at $\tilde{r}_{0} \gg 1$, so that we can approximate the force in Eq. (2) by its asymptotic form $\propto 1 / \tilde{r}$. Integration for the uniform bunch yields

$$
\tilde{r}_{p}=\frac{2 \sqrt{\pi}}{k} \frac{\phi_{e}}{2 \pi}
$$

with $k=1,2,3, \ldots$ This model is plotted with red markers in Fig. 4(b). For the case of Gaussian bunch a direct integration is not possible as $\omega_{e}(z)$ is $z$ dependent. We find then

$$
\frac{1}{2} \int_{z_{\text {ini }}}^{z} \sqrt{-\frac{\left[\tilde{r}^{\prime 2}(z)\right]^{\prime}}{\log ^{\prime}[\tilde{r}(z)]}} d z=\phi_{e}(z),
$$

where ()$^{\prime}=\partial() / \partial z$. An inversion of this relation would give the position of the stripe $\tilde{r}$ as a function of $\tilde{r}_{0}$ and $\phi_{e}$, and the extreme point (in the variable $\phi_{e}$ ) of this function $\tilde{r}\left(\phi_{e}, \tilde{r}_{0}\right)$ is an estimate for the location $r_{p}$ of a stripe. The presence of several stripes again stems from a jump of $\pi$ of $\phi_{e}$. More empirically, the simulation data for an intermediate range of $\phi_{e}$ values, $0.4 \lesssim \phi_{e} \lesssim 1$, can be described by

$$
\left|\tilde{x}_{p}\right| \approx 3 \frac{\phi_{e}}{2 \pi}
$$

which is shown by red markers in Fig. 4(a); for larger values of $\phi_{e}$ the stripe distance from the origin grows faster than linearly. After crossing the beam axis the electrons spread out uniformly in all directions, and the peak density decreases inversely with distance $\tilde{r}$, which may explain the shrinking electron density of Fig. 5.

In a dipole field, as the horizontal amplitude $|\tilde{x}|$ increases, the vertical force on the electrons becomes approximately linear over a larger and larger vertical range, 
$|\tilde{y}| \lesssim|\tilde{x}|$. Therefore, an ever greater number of electrons reach the central plane $y=0$ simultaneously. The regions of increased density on the horizontal axis correspond to the crossing of $y=0$ by large groups of electrons oscillating in the linear vertical beam field at $\tilde{x}$ positions where the local vertical phase advance $\phi_{l, e}(z, \tilde{x})$ is equal to $\pi / 2$ plus a multiple of $\pi$ (the index $l$ indicates the "local" character of this phase advance, that is local as a function of $\tilde{x}$ ).

In fact this $x$-dependent vertical oscillation can be described analytically, starting from Eq. (3), and recalling that the $\tilde{x}$ coordinate is constant. We find that the local frequency $\omega_{l, e}(z, \tilde{x})$ for small $\tilde{y}$ is given by the formula

$$
\omega_{l, e}(z, \tilde{x})=\omega_{e}(z) \sqrt{2 \frac{1-\exp \left(-\tilde{x}^{2} / 2\right)}{\tilde{x}^{2}}} .
$$

From this relation we can easily determine the $\tilde{x}$-dependent phase advance, and, therefore, the pinch locations $\left(z_{p}, \tilde{x}_{p}\right)$ in the plane $(z, \tilde{x})$, which are located at $\phi_{l, e}\left(z_{p}, \tilde{x}_{p}\right)=$ $\pi / 2+(k-1) \pi$, with $k=1,2,3, \ldots$ More conveniently, using the central linear phase advance $\phi_{e}(z) \equiv \phi_{l, e}(z, 0)$ as independent variable rather than $z$, we can express this relation as

$$
\frac{\phi_{e p}}{2 \pi}=\frac{2 k-1}{4 \sqrt{2}} \frac{\tilde{x}_{p}}{\sqrt{1-\exp \left(-\tilde{x}_{p}^{2} / 2\right)}},
$$

where now the pinch is located at $\left(\phi_{e p}, \tilde{x}_{p}\right)$. In Figs. 4(c) and 4(d), the red markers indicate this analytical prediction for comparison with the simulation results.

Data and analytical curve are in excellent agreement. The region where the vertical motion is approximately linear and, therefore, also the number of "synchronized" electrons crossing the $y=0$ plane at the same time grow in proportion to the distance $|\tilde{x}|$, which may explain the density evolution for a dipole field seen in Fig. 5. The apparent shift of the analytical model with respect to the simulation, in Fig. 4(c), reflects the error of the WKB approximation invoked for finding the phase advance Eq. (5).

\section{E. Summary}

The accumulated phase advance of the linear electron oscillation $\phi_{e}(z)$ determines the spatial structure of the electron pinch, almost independently of the longitudinal bunch profile. The pinch structure is also affected by the presence or absence of a magnetic field. In all cases considered, the high-density stripes are approximately circular rings in the $x-y$ plane. At every phase advance value $\phi_{e}$ equal to a $\pi / 2$ plus a multiple of $\pi$ a new stripe emerges close to the beam axis. Therefore the total number of electron-cloud stripes $N_{s}$ is given by

$$
N_{s}=2 \frac{\sqrt{r_{e} N_{b} \sigma_{z}}}{\sigma_{r}} \xi \frac{1}{\pi}+\frac{1}{2},
$$

where $\xi$ is depending on the longitudinal profile, namely

$$
\xi= \begin{cases}\frac{\sqrt{\pi}}{(2 \pi)^{1 / 4}} \simeq 1.11 & \text { Gaussian } \\ \pi \sqrt{\frac{3}{4}} \frac{5^{1 / 4}}{4} \simeq 1.01 & \text { parabolic } \\ \frac{3^{1 / 4}}{\sqrt{2}} \simeq 0.93 & \text { uniform }\end{cases}
$$

where for the uniform bunch $\sigma_{z}=l_{b} / \sqrt{3}$, and $\sigma_{z}=l_{b} / \sqrt{5}$ for the parabolic bunch. The weak dependence of $\xi$ on the bunch longitudinal profile implies that the number of EC stripes depends mainly on the three parameters $N_{b}, \sigma_{z}$, and $\sigma_{r}$ rather than on the shape of the bunch profile. Therefore, we find that rms equivalent bunches not only produce, for high intensity beams, an equivalent evolution of the rms beam envelopes [22], but also produce an equivalent pinch electron dynamics as the number of EC stripes remains the same.

Note that Eq. (11) seems to have a counterintuitive feature: In fact, it predicts that the shorter the bunch is, the less number of EC stripes are created. This seems counterintuitive as it could be argued that shrinking the bunch length increases the charge density and consequently the number of EC stripes. To further verify Eq. (11) we plot in Fig. 6 the EC stripes for a longitudinally uniform bunch of length $\sigma_{z}=0.0432 \mathrm{~m}$ leaving all other parameters unchanged. Equation (11) predicts three EC stripes which we find also by simulation as shown in Fig. 6.

The physical origin of the stripes is different in the fieldfree and dipole case, which explains why in a field-free region the peak electron density decreases as a stripe shifts outwards, while in a magnetic field the peak electron density grows at larger amplitudes. An analytical function

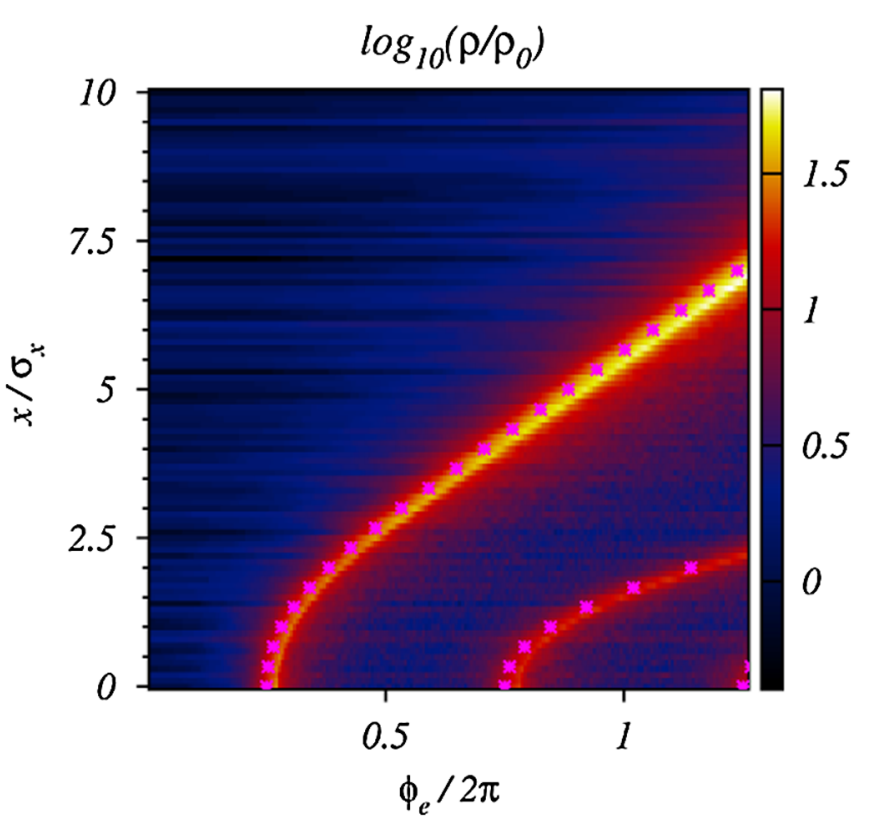

FIG. 6. (Color) Reduction of the EC stripe for shortening the bunch length to $\sigma_{z}=0.0432 \mathrm{~m}$. The number of stripes is correctly predicted by Eq. (11). 
describing the variation of the horizontal stripe position with $\phi_{e}$ for a dipole field is in good agreement with the simulation. The same dependence for the field-free case can be modeled in general analytical terms.

\section{SC \& EC INCOHERENT EFFECTS: DIFFERENCES AND SIMILARITIES}

Space charge is a well-known source of collective effects. It acts most dangerously in a beam at low energy as the beam magnetic self-field scales with energy as $1 / \gamma^{2}$. Typically the radial dependence of the space-charge force is nonlinear, and its strongest gradient occurs in the beam center where, in spite of the fact that the Coulomb force is small, its relative strength with respect to the lattice focusing force becomes strong. The quantity which measures this effect is normally given by the space-charge detuning $\Delta Q_{\mathrm{sc}}$ which measures the tune shift created by the space charge in the transverse center of the beam. The internal nonlinear beam dynamics of high intensity beams is governed by $\Delta Q_{\mathrm{sc}}$, and the dependence of the space-charge detuning on the radial position is essential for all the phenomena of resonance crossing [21]. This effect plays a main role in the dynamics of the incoherent effects. It should also be noted that the space charge Coulomb force acts continuously on the particle beam itself, and it is largely affected by the optics properties of the accelerator lattice where the beam is stored. Waists of beam envelope enhance locally the space-charge force, which create a distribution of nonlinearities along the lattice exciting a space-charge induced resonance driving term function of the lattice structure. This originates the so-called structure resonances.

Though the effect of a pinched electron cloud on the proton or ion beam shows considerable similarity to the effect of the space-charge force, several important differences can also be noticed. First, we observe that the electrons are localized in some part of the accelerator and do not follow the main beam. Therefore, the Coulomb force produced by the electrons is in first order independent of the energy of the main beam, and, hence, the EC effects do not scale with the beam energy in the same way as for SC. In fact, a shrinking beam size at higher beam energy will increase the forces acting between the ion beam and the EC, and therefore incoherent EC effects might get worse at larger beam energy, which would be exactly the opposite to the SC case. Second, the force exerted by the pinched electrons on the proton or ion bunches is again nonlinear, but its largest gradient does not necessarily occur at the transverse beam center as in the case of SC, due to the complicated structure of the electron pinch. For pinched electrons in a field-free region, the symmetry of the electron dynamics partly compensates the transverse electric field at $x \sim y \sim 0$ after the pinch has taken place. However, the effect of one electron-cloud stripe can again be characterized by its effect on the main beam in terms of an induced shift of the transverse oscillation frequencies. Therefore we consider $\Delta Q_{\mathrm{ec}}$, the detuning at the pinch (i.e., at $z=z_{p}, x \simeq y \simeq 0$ ), as a key parameter characterizing the effect of the pinch and of the EC stripes emerging from it. Note that the origin of this detuning is different from that of space charge. In fact the regions of the accelerator where an electron cloud and therefore the pinch occurs can be (but need not be) highly localized. In such a case, the detuning originating from localized ECs depends mainly on the local optics in the regions where the EC is formed (and on the bunch spacing in a bunch train as far as the process of EC formation is concerned), and it is almost independent of the optics between EC locations, while SC always implies a continuously distributed Coulomb effect.

In spite of these differences there is an important similarity between the effects of space charge and electron cloud for bunched particles: a bunch particle experiences a tune shift which is dependent on its position along the bunch. Therefore the space-charge driven tune modulation induced by the longitudinal motion is seen also for a beam affected by the electron cloud.

Clearly, the periodic crossing of a resonance created by the bunch intrinsic Coulomb forces or by the external pinched electron cloud has a different impact on the particle dynamics of trapping and scattering into resonances.

The final results in terms of halo formation, emittance growth, and beam loss depend on the detailed structure of the amplitude-dependent detuning. As discussed in Sec. IID, in the bunch reference frame the structure of the EC density assumes quite a complex form during the pinch process, which makes its effect on the main beam dynamics in long-term storage particularly difficult to assess.

We present next a direct comparison of the SC incoherent effect with the EC-induced incoherent effect. In order to compare SC and EC incoherent effects, we model the beam dynamics in a constant focusing lattice, and, for the sake of simplicity and to avoid spurious results, we consider two special "frozen" models, one for the SC, and the other for EC. These frozen models consider EC or SC forces which depend on the transverse and longitudinal position inside the bunch, but which do not evolve with the evolution of the beam size or beam intensity. This approximation allows long-term tracking without spurious artifacts from the breaking of symplecticity or numerical noise [20,23]. As long as there is no substantial modification of the beam characteristics, such as for the formation of a weakly populated halo, or for small beam loss, this model can be used in good approximation. In case of substantial beam loss, issues of self-consistency in the numerical modeling should necessarily be addressed. An attempt at including the self-consistency in frozen models was reported in Ref. [24].

Note that the complexity of the EC-induced detuning as explored in Refs. [11,15] does not allow a simplified 
modeling of the detuning as a function of the particle amplitude; we therefore do not attempt here to model the effect, but rather rely on frozen models which automatically incorporate the complexity of the several EC structures affecting the proton bunch.

\section{A. Model of space charge}

We consider a stationary bunched beam, where the particle distribution forms a 3D Gaussian distribution:

$$
\rho(x, y, z) \propto \exp \left[-\frac{x^{2}+y^{2}}{2 \sigma_{r}^{2}}-\frac{z^{2}}{2 \sigma_{z}^{2}}\right] .
$$

We assume that $\sigma_{r} / \sigma_{z} \ll 1$ so that locally the bunched beam is similar to a coasting beam. Then the SC field can be found analytically [6] as

$$
E_{x}(x, y, z)=K_{\mathrm{sc}} e^{-\left[z^{2} /\left(2 \sigma_{z}^{2}\right)\right]} \frac{1}{r^{2}}\left[1-e^{-\left[r^{2} /\left(2 \sigma_{r}^{2}\right)\right]}\right] x,
$$

where

$$
K_{\mathrm{sc}}=2 \frac{\sigma_{r}^{2}}{R_{\mathrm{acc}}^{2}}\left(2 Q_{x 0} \Delta Q_{\mathrm{sc}}\right),
$$

with $R_{\text {acc }}$ denoting the circumference divided by $2 \pi$ and $Q_{x 0}$ the bare tune of the accelerator. $\Delta Q_{\mathrm{sc}}$ is the spacecharge detuning at $x \simeq y \simeq z \simeq 0$. The equation of the electric field for $E_{y}$ is obtained substituting $x$ with $y$ in the right-hand side of Eq. (14). Note that the units of this equation are chosen so that the equation of motion for one particle is given by

$$
x^{\prime \prime}+\left(\frac{Q_{x 0}}{R_{\mathrm{acc}}}\right)^{2} x=E_{x}(x, y, z) \quad z^{\prime \prime}+\left(\frac{Q_{z 0}}{R_{\mathrm{acc}}}\right)^{2} z=0,
$$

where $Q_{z 0}$ is the longitudinal tune. We use the above equations to simulate the motion of particles in a bunch in the presence of space charge. The numerical integration is obtained by applying the SC force in $N$ kicks per turn of integrated strength $E_{x}(x, y, z) \Delta s$, where $\Delta s$ is the longitudinal distance between two consecutive SC kicks. Clearly the choice of the number of SC kicks characterizes the accuracy of the numerical integration. Typically the number of kicks chosen per betatron wavelength is $20 \div 40$ for a detuning of $\Delta Q_{\mathrm{ec}} \sim 0.1$. The finite number of SC kicks creates an error, or shift in the calculated numerical detuning. When using $20 \div 40$ kicks per betatron wavelength, we obtain a tune shift which is less than $1 \%$ from the theoretical exact value.

\section{B. Model of EC stripes}

\section{Model of one EC stripe}

Based on the discussion of Sec. IID, we model the electron pinch structure assuming rotational symmetry of the electron cloud both in a field-free region and in a dipole. We also assume that all the factors which contribute to the electron-cloud formation remain invariant at each passage of a beam bunch. As stated in the Introduction, the case of EC buildup in a bunch train is not treated here. Essential for this modeling is the assumption that the dynamics of the pinch repeats itself at each bunch passage. This allows treating the phenomena of the electron pinch and stripe formation on the beam by a frozen model of the force created by the electrons. This assumption is valid for all cases where the effect of the electrons on the proton beam does not cause a substantial modification of the beam particle distribution.

With these assumptions, one EC stripe is modeled by an electron density of the form

$$
\rho(r)= \begin{cases}f\left(r, r_{p}\right) & \text { if } 0<r<r_{p} \\ 0 & \text { if } r_{p}<r,\end{cases}
$$

if $0<r_{p}<\Delta R$, and

$$
\rho(r)= \begin{cases}0 & \text { if } 0<r<r_{p}-\Delta R \\ f\left(r, r_{p}\right) & \text { if } r_{p}-\Delta R<r<r_{p} \\ 0 & \text { if } r_{p}<r\end{cases}
$$

if $r_{p}>\Delta R$. Here $f\left(r, r_{p}\right)$ is the electron density function within the stripe; $r_{p}$ here represents the transverse amplitude of the stripe experienced by a bunch particle at longitudinal position $z$ with respect to the bunch center; $\Delta R$ is the transverse stripe size. The dependence of $r_{p}$ on $z$ is discussed in Sec. II D. This model extends the previously studied one-dimensional sheet model presented in Ref. [15] and more closely approximates the real situation. The electric field created by the electrons is computed with the assumption that the longitudinal extent of the EC stripe is long enough to be seen by passing particles in the bunch as a "cylindrical sheet": this condition is fulfilled if the slope of the stripe in the $(z, r)$ plane is small compared with 1 , or $r_{p} / \Delta z_{\mathrm{ec}} \ll 1$, where $r_{p}$ is the radial position of the stripe and $\Delta z_{\mathrm{ec}}$ its longitudinal distance from the location of the pinch. The electric field of one stripe, computed from Gauss' law, now assumes the forms reported in Fig. 7, where we again characterize the strength of the pinch in terms of the maximum detuning $\Delta Q_{\mathrm{ec}}$ produced at the location of the maximum electron density, here occurring for beam particles with $r<\Delta R$ at the location where the pinch forms $\left(r_{p}=0\right)$. In analogy to the space-charge case we define an EC-induced "integrated perveance" $K_{\text {ec }}$ which we relate to the electron-cloud-induced tune shift on axis. For the sake of simplicity we choose $f\left(r, r_{p}\right)$ constant in $r$ for the region where $r_{p}>\Delta R$. The dependence on $r_{p}$ is taken so as to preserve the longitudinal electric charge density (i.e., the integral $\int_{0}^{\infty} \rho(r) r d r$ ). The integrated perveance $K_{\mathrm{ec}}$ is expressed in terms of the detuning at the location of the pinch starting from the electric field in Fig. 7. The relation reads 


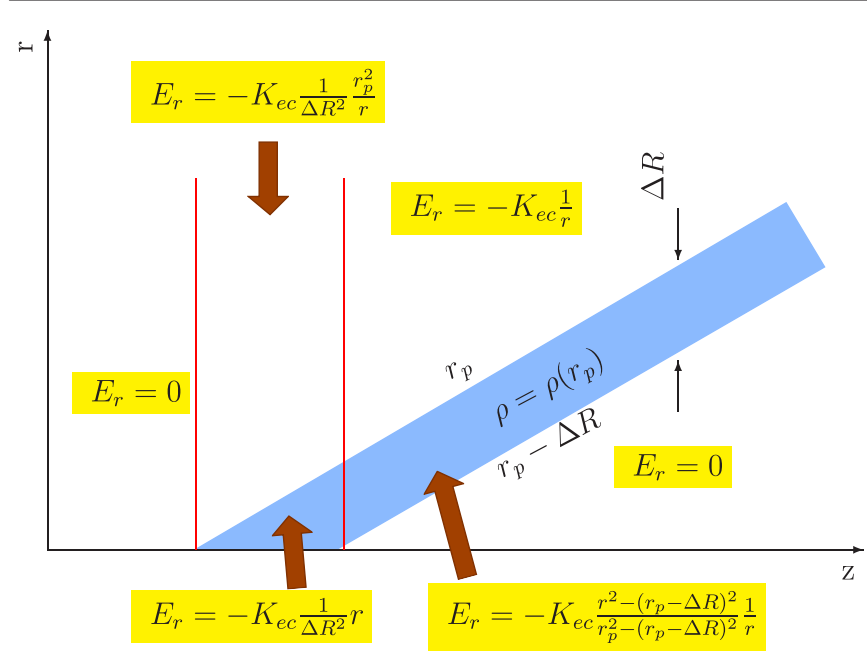

FIG. 7. (Color) The section of the axisymmetric EC stripe divides the plane $(z, r)$ into six regions, in which the analytic form of the EC-induced electric field is explicitly computed.

$$
K_{\mathrm{ec}}=\frac{4 \pi}{\beta_{x}} \Delta Q_{\mathrm{ec}} \Delta R^{2} .
$$

The space-charge detuning is the product of a continuous action of the bunch self-field on one particle of the bunch, while the Coulomb force produced by one EC stripe is interrupted in regions without electron cloud (or with varying electron-cloud density). Note that $E_{r}$ in Fig. 7 has the units of an integrated kick, therefore is dimensionless. This EC kick distributed over an effective length of the EC would create a force which in the equation of motion, Eq. (16), has the dimension $\mathrm{m}^{-1}$.

In the $z-r$ plane, the electron distribution corresponding to the field in Fig. 7 yields a dependence on the distance as $1 /\left[2 r_{p}(z)-\Delta R\right]$ for $r_{p}>\Delta R$, i.e., the effect of the EC stripes is softened at larger radii. Note that, according to the discussion in Sec. II D, it is difficult to relate the properties of the stripes in our model to the maximum induced pinch occurring in a real electron beam. The reason for this is that the pinch simulations exhibit a very peaked density on axis which stems from ignoring the electron-electron space-charge repulsion and the initial velocity spread. A consistent derivation of $\Delta Q_{\mathrm{ec}}$ from the actual on-axis electron density is beyond the scope of the present study.

\section{Modeling of the EC stripes for the full bunch}

Consistently with the discussion in Sec. II D, we take a constant slope $d r_{p} / d z$ as an acceptable approximation for the morphology of the EC stripes. Guided by the discussion of Sec. II D which confirms and extends previous studies on EC-pinch processes for SPS bunches [12,25], we construct a model formed by three electron stripes created along the bunch at the locations of the three pinches $z_{p}=$ $-1 \sigma_{z}, 0.3 \sigma_{z}, 1.5 \sigma_{z}$. Each EC ring has a radial thickness of $\Delta R=1 \sigma_{r}$, and its radial position is $r_{p}(z)=3.33 \times(z-$ $z_{p}$ ) for $z>z_{p}$. This model parameter choice is consistent with the EC pinch in a dipole, whereas for a pinch in fieldfree region $d r_{p}(z) / d z \simeq 2$ (see Fig. 1). In this model a kick is applied at each EC-beam interaction point, e.g., in each dipole magnet (see Sec. IV B), or following a more general kick pattern, e.g., for the purpose of comparing spacecharge effects with EC-induced effects.

\section{Comparison of EC \& SC incoherent effects in the case of a resonance driven by a lattice nonlinearity}

We discuss in this section the effect of the amplitudedependent detuning created by SC or EC on the bunch dynamics in the presence of a lattice-driven resonance. In order to cleanly perform this comparison, we set up a theoretical condition which prevents the EC kick distribution from significantly modifying the lattice-driven resonance. This is accomplished by assigning, artificially, a large number of EC kicks equal to the number of SC kicks, and keeping the same absolute value of the maximum detuning. By doing so we compare the direct effect of the detuning of SC and EC for a lattice resonance of the same strength, and, therefore, we can attribute differences in the beam response (emittance growth or beam loss) directly to the differences of amplitude-dependent detuning for EC and SC. Note that the number of EC kicks is chosen equal to the number of SC kicks for the purpose of comparing emittance growth and beam loss under equivalent conditions. By changing the number of EC kicks, but keeping the same total detuning, we retrieve almost the same amplitude-dependent detuning (unless the number of EC kicks becomes too small), but do so at the price of altering the lattice resonance. To keep the comparison simple, in these simulations we do not include the effect of dispersion and chromaticity, making the modeling independent of the effective bunch length. In fact, the expression of the SC force Eq. (14) or of the EC stripes in the bunch (see Sec. III B 2) is expressed in units of longitudinal length normalized to the rms bunch length, allowing therefore an application to arbitrary bunch lengths. In another, later example (see Sec. IVA) where space charge and chromaticity are included, the effective bunch length must and will be given.

We first consider an example with SPS-like tunes and study the transport of a high intensity axisymmetric bunched beam in the presence of a lattice nonlinearity excited by a single octupole (similarly to what was done experimentally in the CERN-PS experiment [5]).

We set the integrated strength of the octupole according to $K_{3, \text { oct }} \sigma_{r}^{2}=10^{-3}$. The effect of this octupole, beyond the excitation of fourth order resonances, is the creation of a detuning, which in the horizontal plane for a particle with $y=0$ is given by

$$
\Delta Q\left(\frac{X}{\sigma_{r}}\right)=\Delta Q_{\mathrm{oct}, 0} \frac{X^{2}}{\sigma_{r}^{2}},
$$


where $\Delta Q_{\text {oct }, 0}=-\left(\beta_{x} K_{3, \text { oct }} \sigma_{r}^{2}\right) /(32 \pi), X$ is the particle oscillation amplitude $X=\sqrt{\beta_{x} \epsilon_{x}}$, and $\beta_{x}$ the beta function at the location of the octupole. With our setting $\Delta Q_{\text {oct }, 0}=$ $4.2 \times 10^{-4}$. The analysis of the excited resonance is made numerically. Our first simulations contain neither electron cloud nor space-charge forces. The single octupole excites all harmonics, and in order to evaluate the effective stop band of the excited resonances we performed a scan of the beam loss over the $\left(Q_{x 0}, Q_{y 0}\right)$ plane. In this particular simulation we assume a beam pipe of size $3.3 \sigma_{r}$ for the purpose of identifying the relevant resonances via beam losses. At each working point of the scan we compute the survival of $10^{3}$ macroparticles after 300 turns. The simulation results are shown in Fig. 8(a). The longitudinal beam motion is frozen here, and Coulomb forces are absent as we intend to resolve pure nonlinear lattice effects. This picture shows that the resonances $4 Q_{x 0}=105,4 Q_{y 0}=105$, and $2 Q_{x 0}-2 Q_{y 0}=0$ are excited. We then increase the beampipe radius to a larger value of $10 \sigma_{r}$, and simulate the beam loss for $Q_{y 0}=26.136$, and several horizontal tunes in the range $26.1<Q_{x 0}<26.3$ : these results are shown in Fig. 8 (b) for $1.5 \times 10^{5}$ turns. We observe that the resonance $4 Q_{x 0}=105$ is weakly excited and a small emittance growth appears but no beam loss is detected. This is related to the large dynamic aperture with respect to the beam size. The lack of beam loss does not prevent, however, that fourth order islands are created by the single octupole. At the beginning of the tracking, a few particles in the tail of the distribution are found inside these islands leading to the emittance increases. At $Q_{x 0} \simeq 26.137$ the resonance $2 Q_{x 0}-2 Q_{y 0}=0$ is intercepted with significant beam loss because of the reduction of the dynamic aperture.

\section{Pure SC effects}

We now study the response to the high intensity of the bunched beam including the synchrotron motion. We set the space-charge tune spread as $\Delta Q_{\mathrm{sc}}=-0.075$. Note that here, differently from the octupole, the horizontal detuning for a particle with $y=0$ in a Gaussian transverse distribution scales as

$$
\Delta Q\left(\frac{X}{\sigma_{r}}\right) \simeq \frac{\Delta Q_{\mathrm{sc}}}{1+X^{2} /\left(4 \sigma_{r}^{2}\right)},
$$

where again $X$ is the maximum particle transverse amplitude $X=\sqrt{\beta_{x} \epsilon_{x}}$ (see Ref. [6]). The pattern of the tune shift of SC is substantially different from the one induced by the octupole alone. The effects obtained by the SC on the trapping involve particles in the beam center, which otherwise would not be affected by the pure octupole detuning. Again, the dynamics is studied numerically. The effect of space charge (in absence of EC) is obtained by applying 21 equally spaced SC kicks per betatron wavelength, which provides enough computational accuracy for the SC detuning: The numerical error in the detuning, for $Q_{x 0}=26.2$, is smaller than $0.1 \%$. The beam is kept bunched by applying a longitudinal linear focusing force such as to produce a longitudinal tune of $Q_{z 0}=1 / 300$. A partially compensated chromaticity is also included, creating a chromatic tune spread of $\Delta Q_{\mathrm{chr}}=$ \pm 0.015 . The SC effects during long-term beam storage are revealed by performing a tune scan similar to the one made for Fig. 8(b). The results are presented in Fig. 9(a). The beam emittances $\epsilon_{x / y}$ (black/blue) after $1.5 \times 10^{5}$ turns are plotted versus $Q_{x 0}$; in green we depict the beam survival. These results are characteristic of the SC effects (see Ref. [7]), where an emittance growth on the right of the resonance over a region as large as the SC tune spread is formed. The tiny beam loss region with a peak loss of $12 \%$ appears as a consequence of the residual chromaticity and is as large as the chromatic tune spread.

\section{Pure EC effects}

In the next example we ignore SC and consider an equally large maximum detuning of $\Delta Q_{\mathrm{ec}}=+0.075$, now due to EC, at the pinch location on axis. The number of EC kicks is kept the same as in the previous subsection,
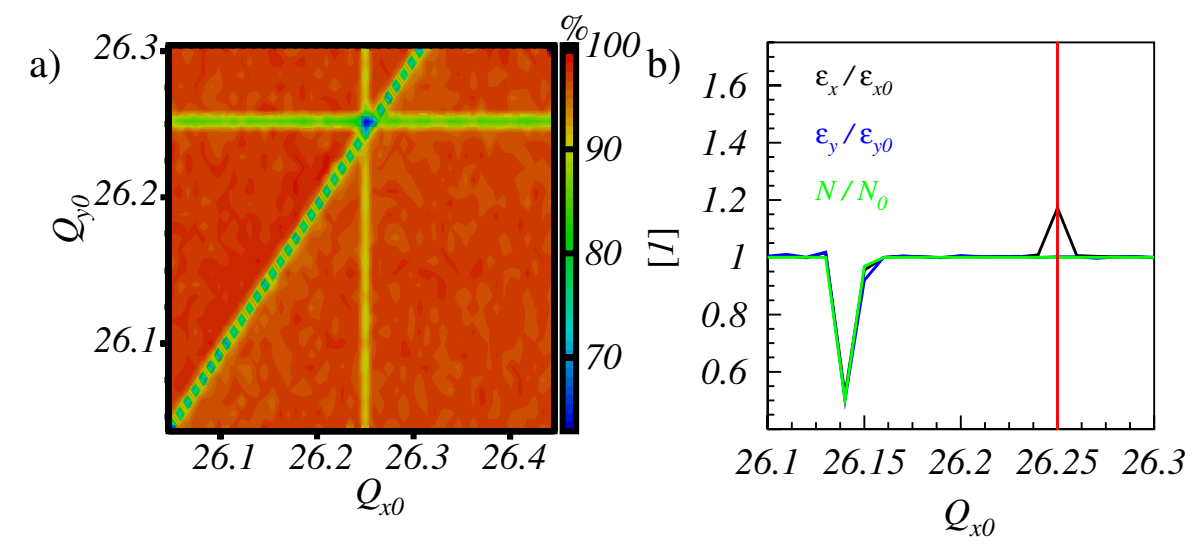

FIG. 8. (Color) Resonance lines excited by a single octupole (left); emittance growth and beam loss for several tunes at $Q_{y 0}=26.136$ (right). 

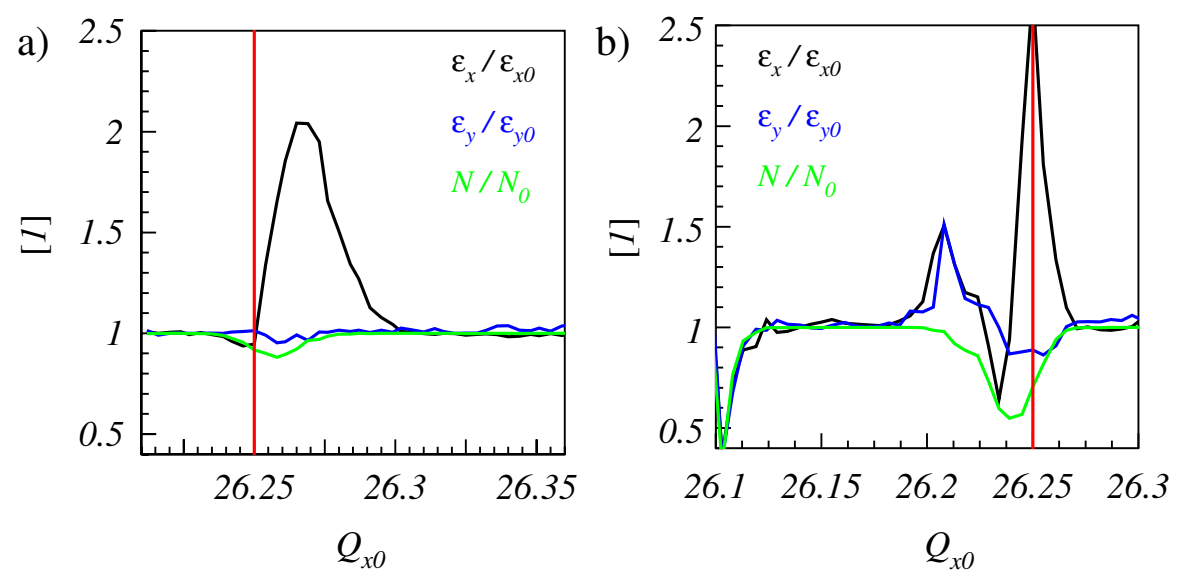

FIG. 9. (Color) Octupolar resonance: space-charge-induced emittance increase and beam loss (left); electron-cloud-induced emittance increase and beam loss (right). In both cases we apply 21 SC or EC "kicks" per betatron wavelength and synchrotron motion is included. In all these simulations $Q_{y 0}=26.1$.

namely, again 21 kicks equally spaced kicks per betatron wavelength: by doing so we remove the effect of the structure resonances. In Fig. 9(b) we plot the same beam quantities as for Fig. 8, but now for the case that the incoherent effects are EC driven. The picture is more complex than the one for SC: Here beam losses are localized on the left of the resonance as a consequence of the positive detuning exerted by the pinched electrons on the strong beam. In the region $26.2 \lessgtr Q_{x 0} \lesssim 26.27$, some beam loss is found as a result of the complex dependence of the detuning on both the transverse and longitudinal amplitude. In the region $26.18 \lesssim Q_{x 0} \lesssim 26.225$ we observe an emittance growth with maximum occurring at $Q_{x 0} \simeq 26.21$ just at the beginning of the beam loss region. This observation is consistent with the previous SC studies which showed that the emittance does not grow when significant beam loss is present [26]. Note, however, that on the fourth order resonance $4 Q_{x 0}=105$, in spite of the beam loss, the horizontal emittance increases by a factor 2.5. We attribute this large emittance growth to the presence of 3 EC rings. The beam loss at $Q_{x 0} \sim 26.1$ is the effect of the coupled fourth order resonance, discussed for Fig. 8.

\section{Discussion}

In this section we compare the effect of space charge and of electron-cloud stripes on the bunch dynamics for longterm storage. Specifically we compare the long-term effect created by trapping by, or scattering off, a resonance when the resonance crossing is caused by the synchrotron motion. We consider a linear lattice augmented by only one octupole which in this study serves to provide the lattice resonance (i.e., the resonance excited by a lattice nonlinear element). The beam response for both cases exhibits some (anti)symmetry due to the opposite signs of the tune shift (Fig. 9). The main difference in the beam response arises from the different amplitude-dependent detuning intro- duced by SC and by EC. An example of this complex dependence was studied for a simpler one-dimensional model of the EC stripe in Ref. [15]: The presence of three EC stripes enhances the detuning at large transverse amplitudes, driving the resonance-induced island even further outward, thereby leading to larger beam loss.

We should mention here that, as shown by Eqs. (20) and (21), the effect of the detuning due to the octupole becomes relevant at large amplitudes. Therefore the migration of islands at large amplitude is controlled by the combined detuning from all sources including the octupole. This type of effect will always be strongly dependent on the detailed features of the nonlinear dynamics in a storage ring.

\section{Comparison of SC \& EC incoherent effects in the presence of a structure resonance}

As discussed at the beginning of this section, the Coulomb force generated by the beam or by the localized electron cloud can create the driving term for a "lattice" resonance (structure resonance). In order to compare SC and $\mathrm{EC}$ in this context, we take the same bunch as described in the previous section, but apply the SC or EC with 105 kicks along the ring so as to excite the fourth order structure resonance. It is convenient to compare the longterm Coulomb incoherent effect from two different sources, but in similar conditions. Again we set the maximum SC detuning $\Delta Q_{\mathrm{sc}}$ or the maximum detuning of $\mathrm{EC}$ at pinch location $\Delta Q_{\mathrm{ec}}$ equally large: $\Delta Q_{\mathrm{sc}}=-\Delta Q_{\mathrm{ec}}=$ -0.075 . We first compare the incoherent effect for the condition of a longitudinally frozen motion, which we can think of as the SC or EC counterparts of Fig. 8. In Fig. 10(a) we plot the emittance growth for a purely SC driven effect for the bunched beam. We find the characteristic asymmetric beam response typical of the SCdominated 2D beams $[27,28]$. The lack of self-consistency plays a minor role as the number of particles beyond $3 \sigma_{r}$ is less than 8\%. For comparison we plot in Fig. 10(b) the 

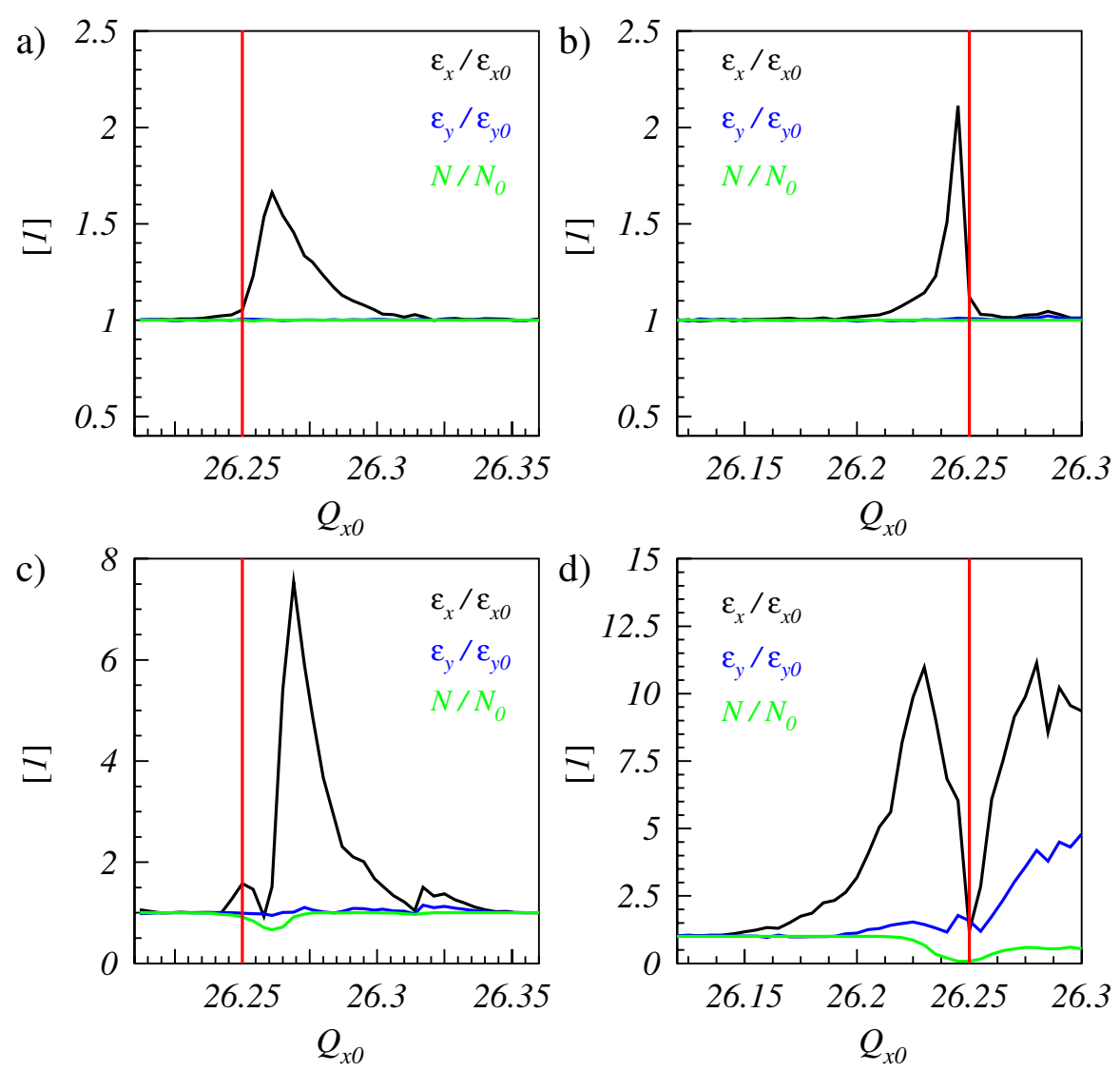

FIG. 10. (Color) Beam response and survival with longitudinally frozen motion (top) pure SC (left), pure EC (right); and including longitudinal motion (bottom) with only SC (left), with only EC (right). In all these simulations $Q_{y 0}=26.1$.

equivalent case of a pure EC incoherent effect. The picture is somewhat the mirrored situation of Fig. 10(a) with respect to the fourth order structure resonance. The difference in emittance increase stems from the EC pinch modeling, which here assumes three EC rings and creates a persistent detuning in the region of the bunch with $z>$ $+1 \sigma_{z}$. The fraction of particles beyond $3 \sigma_{r}$ of the beam is $7 \%$, justifying a frozen model approach. Clearly no periodic crossing of fourth order structure resonance happens in Fig. 10(a) and 10(b) as there is no synchrotron motion. The beam response to the longitudinal motion and the consequence of the tune modulation-via SC or EC tune shift—is visible for both cases in Figs. 10(c) and 10(d). The comparison with Fig. 9 reveals a significant effect which stems, in this example, from the much stronger fourth order driving term compared with the octupolar error used for the simulations of Fig. 8. We address this issue in the next sub section. In Fig. 10(d) the large emittance increase for $Q_{x 0}>26.25$ is a consequence of the structure fourth order resonance $2 Q_{x 0}+2 Q_{y 0}=105$ excited by the EC kicks but absent in Fig. 8.

\section{Remark on the strength of the structure resonance}

The request of the condition $\Delta Q_{\mathrm{sc}}=-\Delta Q_{\mathrm{ec}}=-0.075$ has some unavoidable consequence on the strength of the excitation of the fourth order resonance. We assess this by analyzing the integrated third order component of the EC or SC force. First, we recall that the integrated strength of the octupole applied in our model is

$$
\tilde{K}_{3, \mathrm{oct}}=K_{3, \mathrm{oct}} \sigma_{r}^{2}=10^{-3} .
$$

The tilde $(\tilde{\cdot})$ expresses this quantity when the equations of motion are written in scaled coordinates (i.e., $\tilde{x}=x / \sigma_{r}$, $\tilde{p}_{x}=p_{x} / \sigma_{r}$, etc.). The third order component of the space charge is found by expanding the force in Eq. (14). A straightforward calculation gives as integrated strength of all the third order kicks,

$$
\tilde{K}_{3, \mathrm{sc}}=6 \pi \frac{Q_{x 0} \Delta Q_{\mathrm{sc}}}{R_{\mathrm{acc}}} .
$$

For the EC force, the analysis is more difficult due to the discontinuity of the model taken. However, we can expand the force at the pinch location (where $r_{p}=\Delta R$ ) around $r=r_{p}$. With this approach the electric field is expressed as a power series in $\left(r-r_{p}\right)^{n}$. By taking the third order component and using the definition of $K_{\mathrm{ec}}$ in Eq. (19), we estimate the integrated strength of all EC third order kicks as 


$$
\tilde{K}_{3, \mathrm{ec}}=24 \pi \frac{Q_{x 0} \Delta Q_{\mathrm{ec}}}{R_{\mathrm{acc}}}\left(\frac{\sigma_{r}}{r_{p}}\right)^{2},
$$

where in our model $r_{p}=\Delta R=1 \sigma_{r}$.

Therefore for $Q_{x 0}=26,\left|\Delta Q_{\mathrm{ec} / \mathrm{sc}}\right|=0.075$, and $R_{\mathrm{acc}}=$ $1100 \mathrm{~m}$, we find $\tilde{K}_{3, \text { oct }}=10^{-3}, \tilde{K}_{3 \text {,sc }}=0.033$, and $\tilde{K}_{3, \text { ec }}=$ 0.133: The integrated strength of the "octupolar" component deriving from the space-charge force (i.e., the cubic term) is 33 times larger than the external octupolar error assumed in Fig. 8. The EC integrated kick is 4 times larger than the SC integrated kick. This further explains the large beam response found in Figs. 10(c) and 10(d).

\section{EXAMPLES OF INCOHERENT EFFECTS}

As pointed out, the impact of the incoherent effects generated by high intensity bunched beams or by structured pinched EC requires the consideration of long-term beam storage. For medium energy projects like the SIS100 of the FAIR project [29], long-term diffusion in high intensity bunches should be carefully controlled. The RCS at JPARC [30] will operate in a similar regime, while the JPARC main ring exceeds $10^{4}$ turns of storage time, and for either machine the high intensity incoherent effects should be carefully assessed. In the SPS synchrotron at CERN, the presence of EC is assumed to play a role in the degradation of the bunch lifetime in LHC-type trains of 72 bunches. Reference [31] reports experimental data supporting the interpretation that EC-trapping-related effects induces bunch shortening correlated to beam loss. This experimental evidence finds its SC counterpart in the results of the CERN-PS benchmarking experiment [7], where this effect was directly measured in a dedicated beam study. The complexity of the presence of EC was experienced in RHIC, and an extensive campaign of measurements and countermeasures is described in Ref. [32]: The slow emittance growth at RHIC is suspected to be caused by EC incoherent effects [33]. EC incoherent effects are also of concern for LHC, where many hours of storage for colliding beams in the presence of a possible slow EC-induced diffusion might affect the collider luminosity performance.

\section{A. Application to SC incoherent effects of ion beams in SIS100}

In the SIS100 synchrotron in the FAIR project at GSI [34] bunches of $\mathrm{U}^{28+}$ ions are stored for a time of the order of a second. Controlling radiation damage [35] and containing the negative effects of beam loss on vacuum and magnets-which rely on NEG coating [36] and on a dedicated new halo collimation concept [37]—require a maximum acceptable beam loss of (much) less than $10 \%$ over the total accelerator cycle. We study here the incoherent SC effect during the $1 \mathrm{~s}$ long injection flat-bottom for working point 1 (WP1): $Q_{x / y}=18.84 / 18.73$. In SIS100 the main lattice nonlinearities are the multipole field errors in the superconducting ( $S C$ ) dipoles, conveniently described via an elliptic coordinate transformation [38,39], and the multipole field errors of the $S C$ quadrupoles taken from [40]. Chromatic correction sextupoles are ignored. The purely systematic multipoles yield a short term dynamic aperture $\left(10^{3}\right.$ turns) of $4.8 \sigma$ for a reference beam of $8.75 \mathrm{~mm}$ mrad $\mathrm{rms}$ emittance with the beam magnetic rigidity at injection of $18 \mathrm{Tm}$. A random fluctuation with maximum amplitude of $\pm 30 \%$ is added to the systematic multipoles of the $S C$ dipoles [41]. In this modeling we also take into account a possible residual closed orbit distortion (COD) after correction of $1 \mathrm{~mm}$ vertical $\mathrm{rms}$ (and $1.6 \mathrm{~mm}$ horizontally) which, through the field-error feed-down, yields an average DA of $3.3 \sigma$ with a variance of $0.21 \sigma$. The statistical effect of the residual closed orbit is analyzed over a wide range of working points in Fig. 11(a). For each WP we plot $\left(\langle\mathrm{DA}\rangle-3 \sigma_{\mathrm{DA}}\right)$ by evaluating ten random
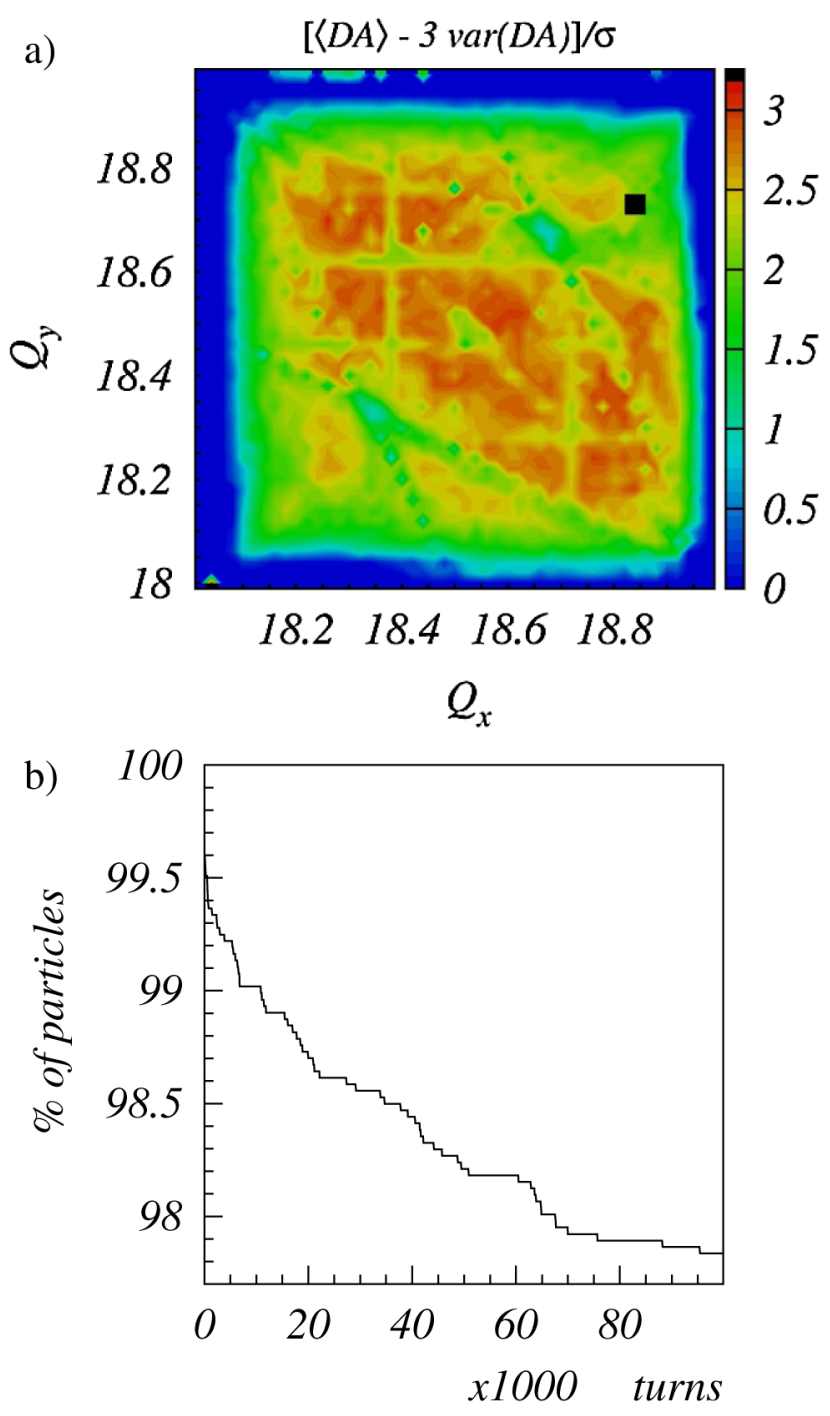

FIG. 11. (Color) SIS100 dynamic-aperture scans with reference random errors (top). Black marker: proposed working point WP1; beam 2 for the standard error case (bottom). 

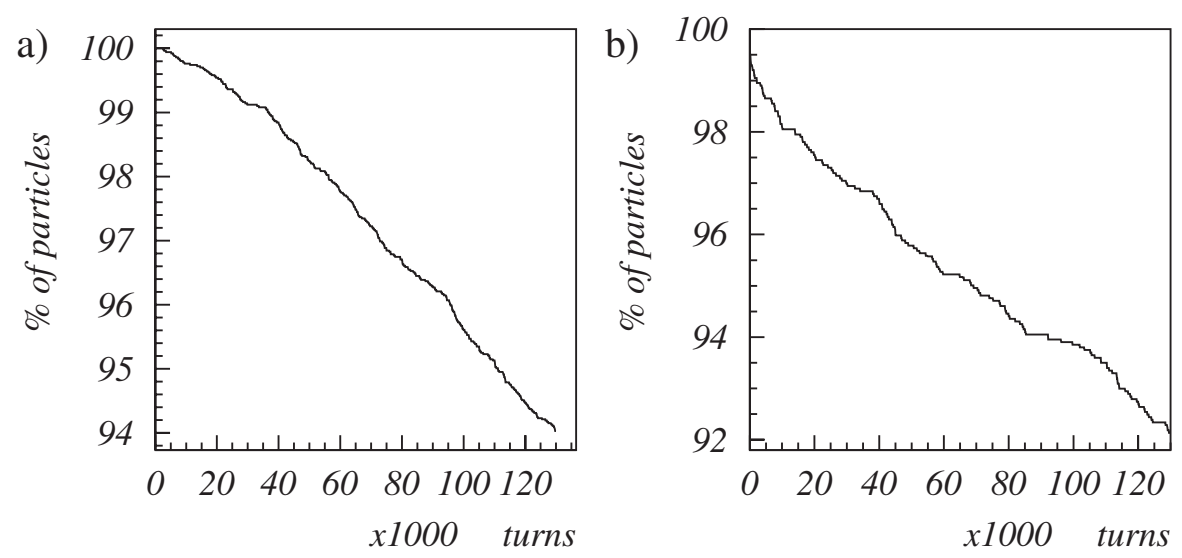

FIG. 12. SIS100 beam loss with space charge for beam 1 (left) and beam 2 (right) for an intensity of $3 \times 10^{11}$ ions.

seeds of $S C$ dipole errors as well as of the $1 \mathrm{~mm}$ vertical rms closed orbit. We model the bunched beam by a Gaussian transverse distribution truncated at $2.5 \sigma$ in amplitude as the result of a controlled beam shaping during transfer from SIS18 to SIS100.

Two sets of reference emittances $(2 \sigma)$ are defined: Beam 1: $\epsilon_{x / y}=35 / 15 \mathrm{~mm}$ mrad (edge at $2.5 \sigma<\mathrm{DA}=$ $3.1 \sigma$ ), which assumes no dilution within the SIS18 acceleration cycle; beam 2: $\epsilon_{x / y}=50 / 20 \mathrm{~mm} \mathrm{mrad}$ (edge at $2.98 \sigma<\mathrm{DA}=3.1 \sigma$ ), which allows for some dilution getting closer to the dynamic-aperture limitation, but reducing the SC tune shift. Including all systematic and random terms so far discussed we explored 27 error seeds consistent with the standard $1 \mathrm{~mm}$ vertical rms COD. The beam loss was computed over $10^{4}$ turns, and we singled out a "standard error case" with the moderately pessimistic beam survival of 99\% [Fig. 11(b) extends prediction till $10^{5}$ turns]. Simulation results for the standard error case including chromaticity show that up to $10^{5}$ turns $(0.6 \mathrm{~s})$ the beam 1 exhibits a beam loss up to about $1 \%$, while for beam 2 we find $6 \%$ beam loss. We then evaluated the effect of the chromaticity in a bunched beam with rms momentum spread of $\delta p / p=5 \times 10^{-4}$ consistent with a bunch length of $\pm 90^{\circ}$ (bunching factor of 0.33) and linear synchrotron period of 233 turns (rf voltage of $53 \mathrm{kV}$ if SC is ignored). Simulations with SC are made with MICROMAP including all previously discussed effects for the standard error case. The space charge is computed with a frozen model, which incorporates the local beam size, as defined by the beam optics [42].

For the nominal total intensity of $6 \times 10^{11}$ of $\mathrm{U}^{28+}$ ions in eight bunches the SC horizontal/vertical tune spread is $-0.31 /-0.47$ for beam 1 and $-0.21 /-0.34$ for beam 2 . In Figs. 12(a) and 12(b) we present results for beam 1 and beam 2 after $1.2 \times 10^{5}$ turns ( $0.7 \mathrm{~s}$ storage) at half the nominal intensity, which helps avoiding the half-integer resonance. In comparison with the case without $\mathrm{SC}$ the beam 1 is dominated by $\mathrm{SC}$ as losses increase from $1 \%$ to $6 \%$ when space-charge forces are included. For beam 2 the loss is dominated by the DA and chromaticity, and adding SC only leads to an increase of the loss from $6.5 \%$ to $8 \%$. The SC-dominated loss for beam 1 at half nominal intensity can be understood as a result of the periodic crossing of the tune footprint with the third order error resonance $2 Q_{x}+Q_{y}=56$, possibly also with $3 Q_{y}=56$. The nominal intensity for the same set of parameters (and the same error set) results in a more than proportional increase of the loss. At maximum intensity many more particles cross the resonance $2 Q_{y}+Q_{x}=56$ and become candidates for loss. We have therefore investigated an alternative working point: $\left(Q_{x}, Q_{y}\right)=(18.84,18.40)$, which is exposed to the apparently weaker third order resonance $Q_{x}+2 Q_{y}=56$. Results for beam survival over the full cycle are obtained by the summing the beam losses accumulated by each of the eight bunches, which are injected over a one-second period. In this process the first bunch is stored for $1 \mathrm{~s}$, the second for $0.875 \mathrm{~s}$, the third for $0.75 \mathrm{~s}$, and so on. Each of these bunches will have the same time survival pattern just time shifted according to the respective injection time. As simulations show that, to a good approximation, the beam loss pattern is linear in time (see Fig. 12), we find that the total beam loss over $1 \mathrm{~s}$ relative to the total injected ions is just half of the relative beam loss for the first injected bunch. The beam survival for the full cycle is presented in Table I.

The loss is improved for full intensity in the alternative working point, but slightly worse for half intensity, possibly because of the proximity of the line $Q_{x}+2 Q_{y}=56$. It should be noted here that the simulation model employed

TABLE I. Beam survival averaged over a full SIS100 cycle for two different working points in tune space.

\begin{tabular}{lcccc}
\hline \hline WP & \multicolumn{2}{c}{$(18.84,18.83)$} & \multicolumn{2}{c}{$(18.84,18.40)$} \\
\hline$\epsilon_{x} / \epsilon_{y}$ & $35 / 15$ & $50 / 20$ & $35 / 15$ & $50 / 20$ \\
Ions/cycle $6 \times 10^{11}$ & $75 \%$ & $78 \%$ & $87 \%$ & $86 \%$ \\
Ions/cycle 3 $310^{11}$ & $97 \%$ & $96 \%$ & $95 \%$ & $91 \%$ \\
\hline \hline
\end{tabular}


in this study lacks dynamical self-consistency. This is not expected to matter for losses at or below the few percent level, but for larger losses inclusion of full self-consistency (e.g., updating the SC force as a consequence of losses) could easily enhance or diminish the loss rate.

\section{B. Exploratory discussion of EC incoherent effects in RHIC and LHC}

RHIC has experienced an incoherent emittance growth attributed to electron clouds [33]. We apply the model of $\mathrm{EC}$ rings resulting from the pinched $\mathrm{EC}$ to one of the two rings of RHIC, the blue ring [43]. In a smooth focusing lattice with a length equal to that of the RHIC blue ring, we introduce 144 EC kicks, one at the position of each long

a)

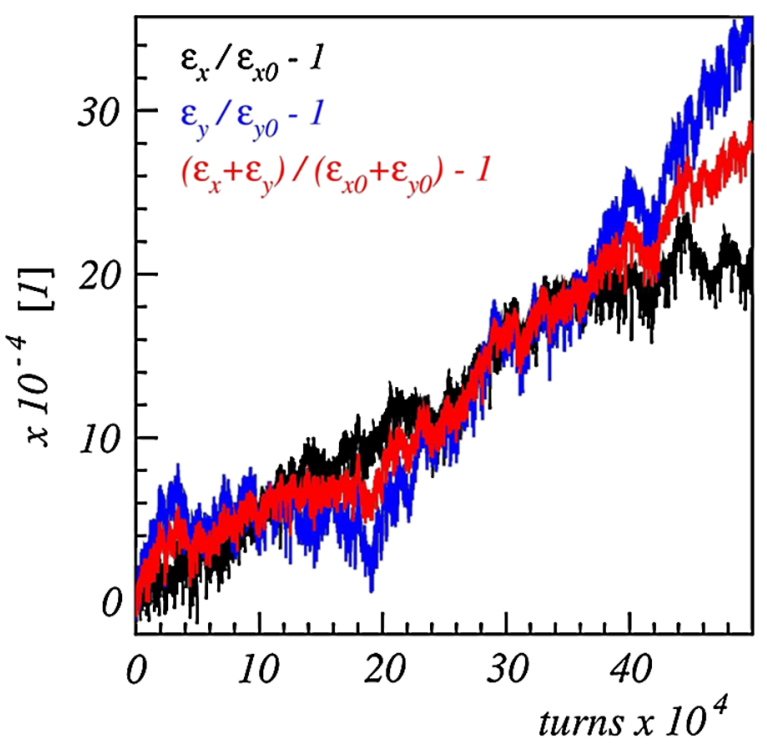

b)

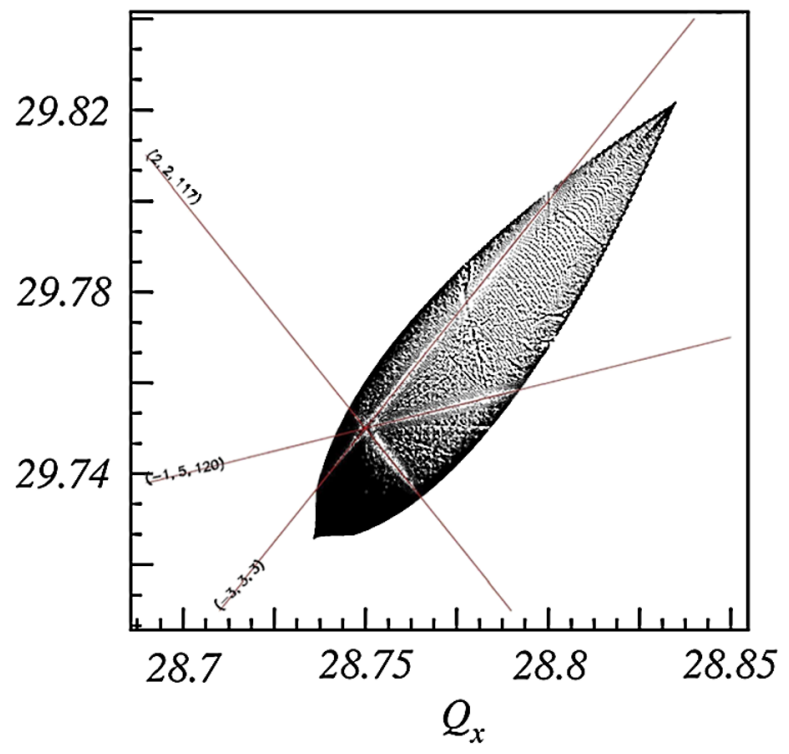

FIG. 13. (Color) RHIC beam emittance evolution versus turns for $\Delta Q_{\mathrm{ec}}=0.03$ (top); frequency map for RHIC for a beam with $\Delta Q_{\mathrm{ec}}=0.1$ (bottom). dipole, because the dipole magnets are considered to be the primary locations for the EC. In doing so, the structure of EC kicks in our model preserves the location of dipoles as in the real lattice geometry, therefore exciting a correct web of EC-induced structure resonances. In order to display only the effect arising from EC, we here again neglect the effect of dispersion and chromaticity. The model of RHIC has tunes $Q_{x}=28.735, Q_{y}=29.725$, and the integrated EC incoherent detuning is taken as $\Delta Q_{\mathrm{ec}}=0.03$ [Fig. 13(a)]. In Fig. 13(b) the main structure resonances excited by the localized electron cloud are found with a frequency map: $-Q_{x 0}+5 Q_{y 0}=120, \quad 2 n Q_{x 0}+$ $2 n Q_{y 0}=117 n,-3 n Q_{x 0}+3 n Q_{y 0}=3 n$, with $n$ integer.
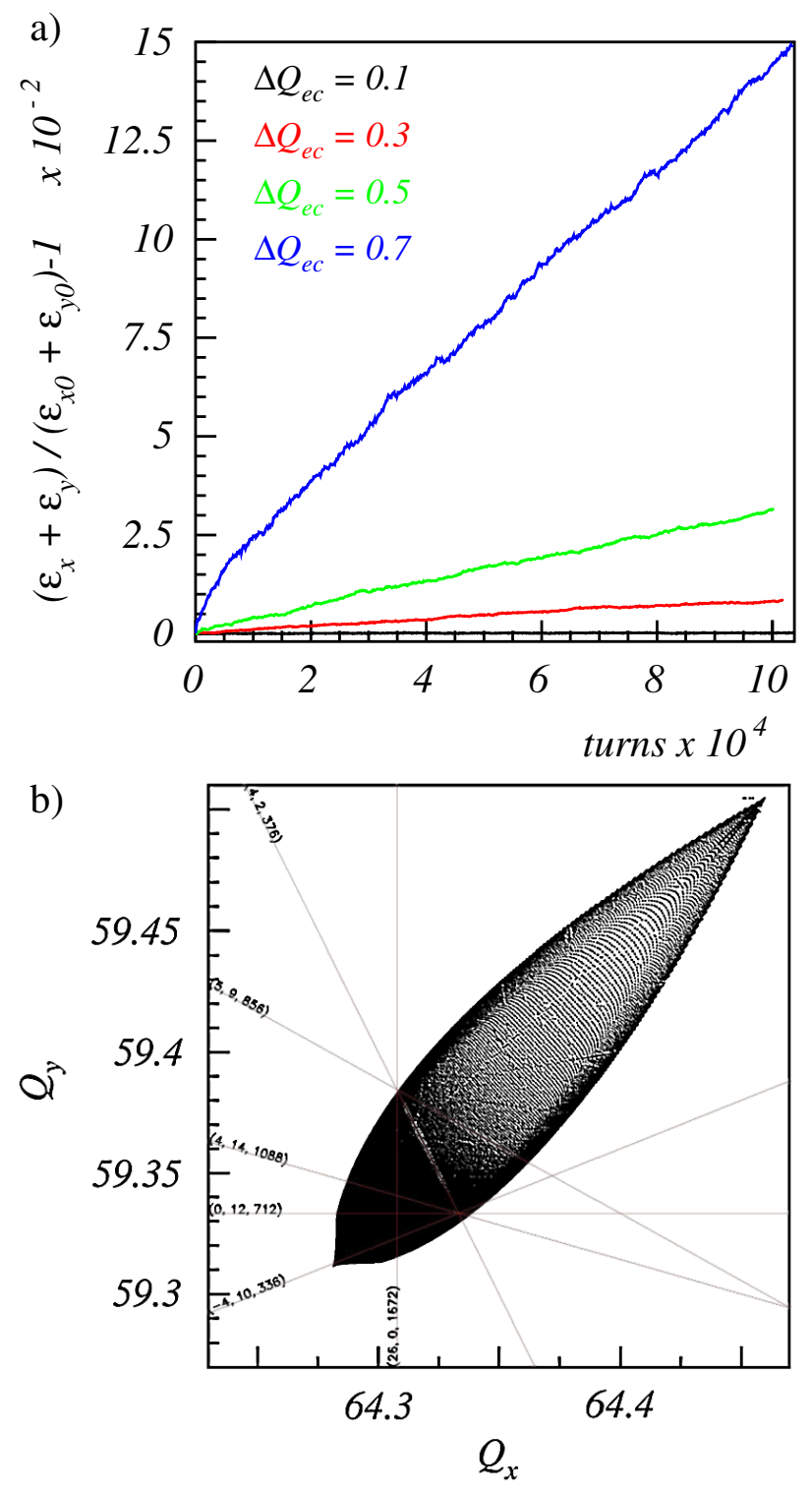

FIG. 14. (Color) LHC beam emittance evolution versus time for $\Delta Q_{\mathrm{ec}}=0.1$ (top); frequency map for LHC for a beam with $\Delta Q_{\mathrm{ec}}=0.18$ (bottom). 
The test particles are taken at $z=-0.73 \sigma_{z}$ and the longitudinal motion is kept frozen. As Fig. 13(b) shows, the ECinduced tune spread causes the crossing of several resonances.

The effect on the dynamics is shown in Fig. 13(a) where a slow emittance increase is found over $5 \times 10^{5}$ of turns. For Fig. 13(a), we find that the average emittance growth has a gradient of $\Delta \epsilon_{\mathrm{av}} / \epsilon_{x 0} \sim 5.6 \times 10^{-9} /$ turn, which at a beam energy of $\gamma=10.52$ for Au ions yields $\Delta \epsilon_{\mathrm{av}} / \epsilon_{x 0} \sim$ $1.5 /$ hour. Clearly, this result is sensitive to the parameter $\Delta Q_{\mathrm{ec}}$ which is chosen here as 0.03 . For weaker values the dynamics of resonance crossing is changed in terms of resonance overlapping, and resonance strength (as these resonances are structure type and their strength is directly proportional to the tune shift): both these factors affect the trapping/scattering regimes, and, therefore, change the diffusion rate.

We finally apply the pinched EC dynamics to the LHC, again applying one EC kick at each of the 1152 LHC main dipoles set up with the correct geometrical, or rather optical, structure of the real machine and tracking the beam in smooth focusing approximation to gain in computational speed. Once again we neglect the effect of chromaticity and dispersion. Here a single bunch is composed of $10^{4}$ macroparticles which are tracked in the absence of chromatic effects through a fully linear lattice. The LHC tunes are $Q_{x}=64.28, Q_{y}=59.31, Q_{z}=1 / 168$, and the LHC has an eightfold symmetry. The integrated EC detuning is, somewhat arbitrarily, set to the values $\Delta Q_{\mathrm{ec}}=0.1,0.3,0.5$, and 0.7 as possible realistic examples for the incoherent EC detuning. The beam evolution is illustrated in Fig. 14(a), showing different average beam responses $\left[\left(\epsilon_{x}+\right.\right.$ $\left.\left.\epsilon_{y}\right) /\left(\epsilon_{x 0}+\epsilon_{y 0}\right)-1\right]$ as a function of $\Delta Q_{\text {ec }}$. The frequency map in Fig. 14(b), obtained for $\Delta Q_{e c}=0.18$ keeping the test particles at $z=-0.73 \sigma_{z}$, reveals that the EC structure resonances $5 Q_{x 0}+9 Q_{y 0}=856,4 Q_{x 0}+$ $2 Q_{y 0}=376$, and $4 Q_{x 0}+14 Q_{y 0}=1088$, together with the EC-induced tune modulation, are responsible for the emittance growth in Fig. 14(a).

\section{CONCLUSION AND OUTLOOK}

We have shown that for both space charge and electron cloud the long-term beam response to incoherent resonance excitation and resonance crossing is a possible source of slow emittance growth. In this respect SC \& EC incoherent effects have similar features. We have evaluated the relevance of these incoherent effects for SIS100, and conducted an exploratory study for RHIC and LHC. The long-term predictions for incoherent SC effects are presently better understood than the corresponding effects for EC, and they are better experimentally benchmarked. EC incoherent effects need further studies and dedicated beam experiments in order to confirm analytical and simulation models for the long-term beam evolution.

\section{ACKNOWLEDGMENTS}

We thank G. Arduini, E. Benedetto, E. Métral, K. Ohmi, K. Oide, G. Rumolo, and the late F. Ruggiero for helpful discussions.

[1] J. Laslett, in Proceedings of the Summer Study on Storage Rings, Brookhaven, 1963 (BNL, Brookhaven, NY, 1963), pp. 324-367.

[2] I. Hofmann, Phys. Rev. E 57, 4713 (1998).

[3] I. Hofmann et al., Phys. Rev. ST Accel. Beams 6, 024202 (2003).

[4] R. Baartmann, in Proceedings of the Workshop on Space Charge Physics in High Intensity Hadron Rings, AIP Conf. Proc. No. 448 (AIP, New York, 1998), p. 56.

[5] G. Franchetti et al., Phys. Rev. ST Accel. Beams 6, 124201 (2003); E. Métral et al., Nucl. Instrum. Methods Phys. Res., Sect. A 561, 257 (2006).

[6] G. Franchetti and I. Hofmann, Nucl. Instrum. Methods Phys. Res., Sect. A 561, 195 (2006).

[7] G. Franchetti et al., in Proceedings of the 33rd ICFA Worksop, AIP Conf. Proc. No. 773 (AIP, New York, 2004), p. 137.

[8] G. Franchetti, in Proceedings of the 2007 Particle Accelerator Conference, Albuquerque, New Mexico, 2007 (IEEE, Albuquerque, New Mexico, 2007), p. 794, TUZAAB02.

[9] G. Rumolo, F. Ruggiero, and F. Zimmermann, Phys. Rev. ST Accel. Beams 4, 012801 (2001); F. Zimmermann, Phys. Rev. ST Accel. Beams 7, 124801 (2004).

[10] K. Ohmi and F. Zimmermann, Phys. Rev. Lett. 85, 3821 (2000).

[11] E. Benedetto and F. Zimmermann, in Proceedings of the 9th European Particle Accelerator Conference, Lucerne, 2004 (EPS-AG, Lucerne, 2004), p. 1834, WEPLT009.

[12] F. Zimmermann and G. Franchetti, in Proceedings of the 11th European Particle Accelerator Conference, Genoa, 2008 (EPS-AG, Genoa, Italy, 2008), p. 3197, THPC089.

[13] E. Benedetto et al., in Proceedings of ECLOUD'04, Napa (CERN Report No. CERN-2005-001, 2004), p. 81; Proceedings of the 9th European Particle Accelerator Conference, Lucerne, 2004 (Ref. [11]), p. 1834.

[14] E. Benedetto, G. Franchetti, and F. Zimmermann, Phys. Rev. Lett. 97, 034801 (2006); K. Ohmi and K. Oide, Phys. Rev. ST Accel. Beams 10, 014401 (2007).

[15] G. Franchetti and F. Zimmermann, in Proceedings of the CARE-HHH-ADP BEAM07 Workshop, Geneva, Switzerland, 2007.

[16] G. Arduini et al., in Proceedings of the 19th Particle Accelerator Conference, Chicago, Illinois, 2001 (IEEE, Piscataway, NJ, 2001), p. 685.

[17] M. A. Furman and A. A. Zholents, in Proceedings of the 18th Particle Accelerator Conference, New York, 1999 (IEEE, New York, 1999), p. 1794.

[18] F. Zimmermann, in Proceedings of Chamonix XI (CERN Report No. CERN-SL-2001-003-DI, 2001), p. 144; Proceedings of the 19th Particle Accelerator Conference, Chicago, Illinois, 2001 (Ref. [16]), p. 666. 
[19] K. Ohmi, Memorandum, November 2002; see http://ababp-rlc-ecloud.web.cern.ch/ab-abp-rlc-ecloud.

[20] E. Benedetto, Ph.D. thesis, Torino Politecnico [CERN Report No. CERN-THESIS-2008-096 CARE-THESIS06-007, 2006].

[21] G. Franchetti, Proceedings of the 11th European Particle Accelerator Conference, Genoa, 2008 (Ref. [12]), p. 2942, THYM02.

[22] Frank J. Sacherer, in Proceedings of the 1971 Particle Accelerator Conference [IEEE Trans. Nucl. Sci. 18, 11051971)].

[23] J. Struckmeier, Phys. Rev. ST Accel. Beams 3, 034202 (2000).

[24] G. Franchetti, I. Hofmann, and S. Machida, in Proceedings of ICAP 2006, Chamonix Mont-Blanc, France, 2006, p. 65, MOA2IS03.

[25] E. Benedetto et al., Proceedings of the 21st Particle Accelerator Conference, Knoxville, 2005 (IEEE, Piscataway, NJ, 2005), p. 1344.

[26] G. Franchetti et al., in Proceedings of the 39th ICFA Workshop, 2006, p. 344, THBW01.

[27] I. Hofmann, G. Franchetti, and A. V. Fedotov, in Proceedings of the 20th ICFA Workshop, AIP Conf. Proc. No. 642 (AIP, New York, 2002), p. 248.

[28] A. V. Fedotov and I. Hofmann, Phys. Rev. ST Accel. Beams 5, 024202 (2002).

[29] Spiller and G. Franchetti, Nucl. Instrum. Methods Phys. Res., Sect. A 561305 (2006).

[30] F. Noda et al., Proceedings of the 21st Particle Accelerator Conference, Knoxville, 2005 (Ref. [25]), p. 2759, MPPE043.

[31] G. Franchetti et al., in Proceedings of LHC-LUMI-06, 2006, p. 192.

[32] W. Fischer et al., Phys. Rev. ST Accel. Beams 11, 041002
(2008).

[33] S. Y. Zhang and V. Ptitsyn, Phys. Rev. ST Accel. Beams 11, 051001 (2008).

[34] P. J. Spiller et al., in Proceedings of the 11th European Particle Accelerator Conference, Genoa, 2008 (Ref. [12]), p. 298, MOPC100; G. Franchetti et al., in Proceedings of the 10th European Particle Accelerator Conference, Edinburgh, Scotland, 2006 (EPS-AG, Edinburgh, Scotland, 2006), p. 2793, THPCH005; SIS100 Technical report, http://www.gsi.de/fair/reports/btr.html.

[35] E. Mustafin et al., Proceedings of the 9th European Particle Accelerator Conference, Lucerne, 2004 (Ref. [11]), p. 1408, TUPLT112; E. Mustafin et al., Proceedings of the 21st Particle Accelerator Conference, Knoxville, 2005 (Ref. [25]), p. 3943, FPAE075.

[36] H. Kollmus et al., Proceedings of the 10th European Particle Accelerator Conference, Edinburgh, Scotland, 2006 (Ref. [34]), p. 1426, TUPCH174; A. W. Molvik et al., Phys. Rev. Lett. 98, 064801 (2007).

[37] C. Omet, Proceedings of the 11th European Particle Accelerator Conference, Genoa, 2008 (Ref. [12]), p. 295.

[38] P. Akishin, E. Fischer, and P. Schnizer, 3D field calculations for the SIS100 dipoles, 2007, GSI internal note.

[39] P. Schnizer, B. Schnizer, P. Akishin, and E. Fischer, Nucl. Instrum. Methods Phys. Res., Sect. A 607, 505 (2009); F. Revuelta Peña and G. Franchetti, GSI Report No. ACC_note-2008-001, 2008.

[40] A. Kovalenko (private communication).

[41] P. Spiller (private communication).

[42] A. Orzhekhovskaya and G. Franchetti, in Proceedings of ICAP 2006, Chamonix Mont-Blanc, France, p. 106, TUPPP05.

[43] H. Hahn et al., Nucl. Instrum. Methods Phys. Res., Sect. A 499, 245 (2003). 